University of Rhode Island

DigitalCommons@URI

Open Access Master's Theses

2018

\title{
Modeling Dynamic Social Networks of Children in After-School Care Programs
}

Valerie Ryan

University of Rhode Island, vmryan@uri.edu

Follow this and additional works at: https://digitalcommons.uri.edu/theses

\section{Recommended Citation}

Ryan, Valerie, "Modeling Dynamic Social Networks of Children in After-School Care Programs" (2018). Open Access Master's Theses. Paper 1184.

https://digitalcommons.uri.edu/theses/1184

This Thesis is brought to you for free and open access by DigitalCommons@URI. It has been accepted for inclusion in Open Access Master's Theses by an authorized administrator of DigitalCommons@URI. For more information, please contact digitalcommons-group@uri.edu. 
MODELING DYNAMIC SOCIAL NETWORKS OF CHILDREN IN AFTER-SCHOOL CARE PROGRAMS

BY

VALERIE RYAN

A THESIS SUBMITTED IN PARTIAL FULFILLMENT OF THE

REQUIREMENTS FOR THE DEGREE OF

MASTER OF ARTS

IN

BEHAVIORAL SCIENCE

UNIVERSITY OF RHODE ISLAND

2018 
MASTER OF ARTS THESIS

OF

VALERIE RYAN

APPROVED:

Thesis Committee:

Major Professor Theodore Walls

Lynda Stein

Natallia Katenka

Nasser H. Zawia DEAN OF THE GRADUATE SCHOOL

UNIVERSITY OF RHODE ISLAND

2018 


\begin{abstract}
Problem: There is a lack of work examining children's social networks outside of the classroom and dynamic network analysis with small networks is one way to see how children influence one another socially over time. The current study utilized an existing database of two after-school care programs represented as networks of friendship connections between children in each program. The children were aged 5 to 12 years old and information was collected at three time points on their activity levels, who they were friends with in the program, and other covariates, such as sex, race/ethnicity, and obesity. We examined whether or not children influence one another's activity levels through their direct friendship connections.

Methods: Dynamic social network analyses were deployed using three different models: separable temporal exponential random graph models (STERGMs), stochastic actor-based models to replicate the original analyses, and models based on the work of Kindermann (2007).

Summary: Findings indicate that activity levels are not important when children are forming friendships, but having a friend with a similar level of activity makes a child less likely to end the friendship.
\end{abstract}




\section{ACKNOWLEDGMENTS}

I would like to acknowledge my major professor, Ted Walls, without whom I would not have been able to complete this thesis. From suggesting that I look up social network analysis in a café during my first semester to asking people at a conference if they had network data I could use, Ted has helped me pursue a topic I care about deeply. I would also like to thank my committee members, Lyn Stein and Natallia Katenka, for their support in this process. And I would like to thank Sabina Gesell for allowing me to use her dataset for this project.

I would also like to thank my Mimi, whose jokes and gentle inquiries about my thesis progress during our weekly Skype chats kept me motivated. And I would like to thank my mom; she enjoys my work.

Lastly, I would like to thank my partner, Dan Kral, for always supporting me, making me food every day, and for his willingness to help me troubleshoot coding issues. Without him, my network graphs would not be in color. 


\section{PREFACE}

Manuscript format has been used in the preparation and dissemination of this master's thesis. The manuscript has not yet been submitted for publication. 


\section{TABLE OF CONTENTS}

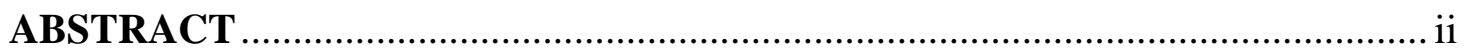

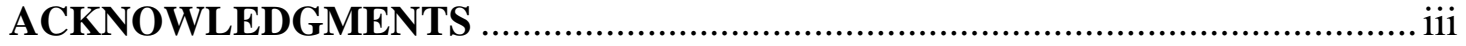

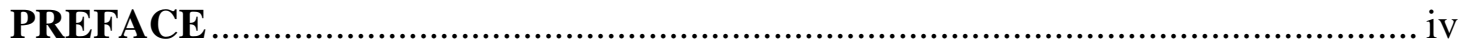

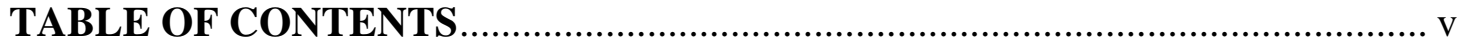

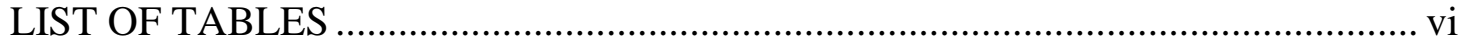

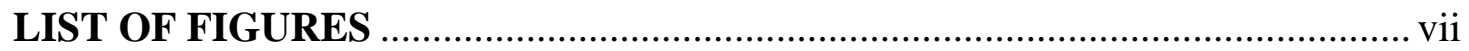

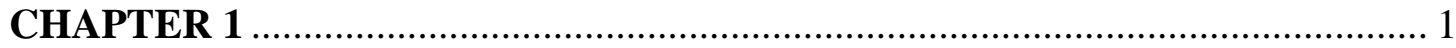

MODELING DYNAMIC SOCIAL NETWORKS OF CHILDREN IN AFTER-

SCHOOL CARE PROGRAMS ............................................................... 1

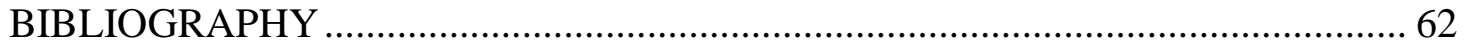




\section{LIST OF TABLES}

TABLE

PAGE

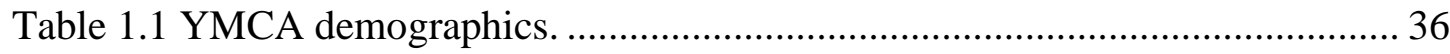

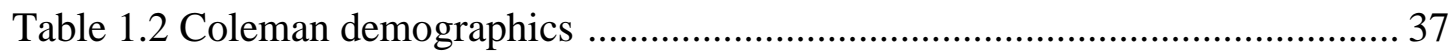

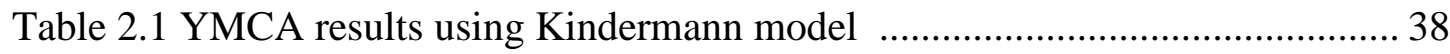

Table 2.2 Coleman results using Kindermann model ................................................ 39

Table 3.1 YMCA results from STERGM full model................................................ 40

Table 3.2 YMCA results from STERGM basic model .............................................. 41

Table 4.1 Coleman results from STERGM full model ........................................... 42

Table 4.2 Coleman results from STERGM basic model ........................................... 43

Table 5.1 YMCA results from Gesell replication - full model …………................. 44

Table 5.2 YMCA results from Gesell replication - basic model ................................ 45

Table 6.1 Coleman results from Gesell replication - full model ................................. 46

Table 6.2 Coleman results from Gesell replication - basic model ............................. 47 


\section{LIST OF FIGURES}

FIGURE

PAGE

Change in Coleman network over time. Node size represents degree (how many

friends a child has), while color represents activity level (dark purple is lowest activity

level, gray is a middle activity level, dark green is highest activity level).

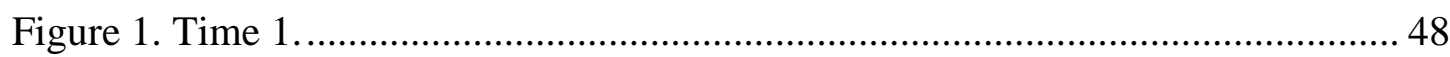

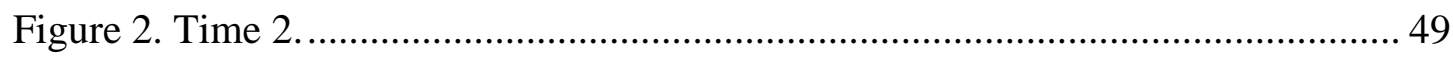

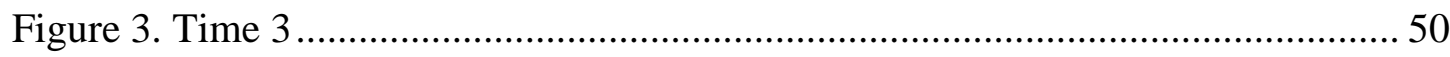

Change in YMCA network over time. Node size represents degree (how many friends a child has), while color represents activity level (dark purple is lowest activity level, gray is a middle activity level, dark green is highest activity level).

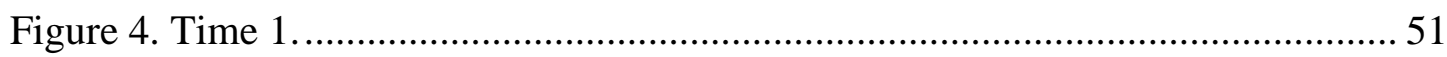

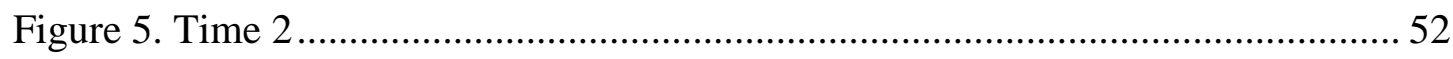

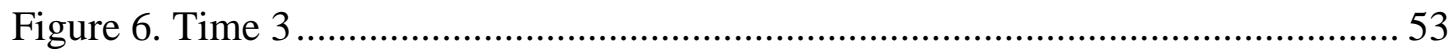




\title{
CHAPTER 1
}

\section{MODELING DYNAMIC SOCIAL NETWORKS OF CHILDREN IN AFTER- SCHOOL CARE PROGRAMS}

\author{
Valerie Ryan ${ }^{\mathrm{a}}$, Theodore A. Walls ${ }^{\mathrm{a}}$, Sabina Gesell ${ }^{\mathrm{b}}$ \\ ${ }^{a}$ University of Rhode Island \\ ${ }^{\mathrm{b}}$ Wake Forest University \\ manuscript will be submitted to Social Psychology
}

Author Note

Valerie Ryan, Department of Psychology, University of Rhode Island Theodore A. Walls, Department of Psychology, University of Rhode Island Sabina Gesell, Department of Social Sciences and Health Policy, Wake Forest University

Data collection was funded by the American Heart Association Clinical Research Grant Program (09CRP2230246).

Correspondence concerning this article should be addressed to Valerie Ryan, Department of Psychology, University of Rhode Island, Kingston, RI 02881

Contact:vmryan@uri.edu 


\begin{abstract}
There is a lack of work examining children's social networks outside of the classroom; dynamic network analysis with small networks is one way to see how children influence one another over time. The current study utilized an existing database of two after-school care programs, represented as networks of friendship connections between children in each program. Information was collected at three time points on childrens' activity levels, their friendships in the program, and other covariates, such as age, sex, race/ethnicity, and obesity. We examined whether or not children influence one another's activity levels through their direct friendship connections by deploying three different network models: separable temporal exponential random graph models (STERGMs), stochastic actor-based models to replicate the original analyses, and models based on the work of Kindermann (2007). Findings indicate that activity levels are not important when children are forming friendships, but having a friend with a similar level of activity makes a child less likely to end the friendship.
\end{abstract}

Keywords: social network analysis, activity level, after school program 
Modeling Dynamic Social Networks of Children in After-School Care Programs Why should we study children's social networks? Children are born into social networks, which consist mostly of family members at infancy. These networks expand over time and begin to include peers and others outside of the family. This process begins as early as age three (Feiring \& Lewis, 1989). As children develop, so do their networks. Major network changes coincide with developmental milestones, such as when a child begins school; their social network increases in size and diversity of relationship types (Feiring \& Lewis, 1989). These network changes could play a key role in a child's development, with their peers influencing their behavior at young ages. These early network interactions have implications for lifespan development. Peer-to-peer influences can be examined through the use of social network analysis, which allows researchers to conduct quantitative research in a familiar way (i.e., by collecting data on individual participants) and then adds another layer: quantifying relationships between participants, beyond looking only at dyads. Using information on the connections ("ties" or "edges") between participants ("actors" or "nodes") we can create a network representation of the relational data and assess how social structure influences research questions of interest (Kolaczyk \& Csárdi, 2014). For example, instead of asking "on average, how much exercise do children with high body mass indices (BMIs) in an after-school program experience over the course of 4 months" we can ask, "do children who are in the same friend group in an after school program experience similar activity levels over time?"

The best way to determine influence effects in networks is by using dynamic network analysis, which models networks longitudinally. Modeling change in a social 
network over time is challenging; methods are currently being developed to improve parameter estimation in this area. This is particularly important for social network analyses using small networks, which can be more difficult to analyze due to issues with model convergence. Improving parameter estimation and model convergence is crucial because children tend to have small social networks (Feiring \& Lewis, 1989). Thus, improving modeling for small networks not only benefits network researchers but also those who study children's networks.

Children's social networks have previously been studied with a focus on adolescent peer-to-peer influences (e.g., DeLay, Ha, Van Ryzin, Winter, \& Dishion, 2016; Valente, Fujimoto, Chou, \& Spruijt-Metz, 2009), specific subpopulations of children, such as children with autism (Anderson, Locke, Kretzmann, \& Kasari, 2016), or within a classroom setting (e.g., Cooc \& Kim, 2017; Golemiec, Schneider, Boyce, Bush, Adler, \& Levine, 2016; Laninga-Wijnen, Ryan, Harakeh, Shin, \& Vollebergh, 2017). These studies miss a crucial component of children's networks: friendships developed outside of school during early- to middle-childhood.

The current study examines two small after-school care networks over time using various dynamic social network analysis methods. We tested three statistical models of network change in social networks of children who participated in one of two after-school programs.

The first set of models come from one of the leading dynamic social network analysis frameworks: stochastic actor-oriented modeling (SAOM). Actor-based modeling assumes that actors are trying to optimize their network positions, which explains their changes in ties and/or behaviors (Gesell, Tedahl, \& Ruchman, 2012). In 
these models, it is assumed that the evolution of networks follows a Markov process, even when networks are measured at discrete time points (Gesell et al., 2012). This process uses only the current state of a network to determine evolution to a future state, rather than including all previous time points to determine network evolution (Snijders et al., 2010). SAOMs have two parts: selection and influence effects. Selection effects refer to factors that influence how children choose their friends, while influence effects include factors that affect a child's outcome behavior, in this case, activity level (Gesell et al., 2012). This model was chosen to replicate the analyses of Gesell et al. (2012), which used the SAOM framework.

The second group of dynamic network models is Separable Temporal Exponential Random Graph Model (STERGM), which add a longitudinal component to traditional exponential random graph models (ERGMs) and was largely developed by Krivitsky and Handcock (2014). ERGMs are similar to regression models used within general linear modeling in non-network statistics (Kolaczyk \& Csárdi, 2014). ERGMs model the probability of a tie being present or absent as a binary random variable, which is conditional on other aspects of the model, such as actor attributes and/or the occurrence of certain structural properties of the network (e.g., how many friends of friends are also friends with each other?). The researcher chooses which attributes and network structures to include in the model - these are analogous to the predictor variables in regression models. Attributes measured in the current study include body mass index (BMI), activity level, age, sex, race, family membership, and school. 
Krivitsky and Handcock's (2014) STERGM is an extension of traditional ERGMs in that it takes observations of the network at discrete time points and models the formation and elimination of ties in the network separately. Krivitsky and Handcock (2014) posit that the prevalence of characteristics of a network at one time point comes from a different source than information about network evolution (duration) over time. Allowing for separate parameterization of prevalence and duration of ties in the after-school program network data is important because the reasons children initially form relationships likely differ from the reasons they end relationships. It's likely that different processes are at work in the formation and dissolution of any social relationship. For example, many friendships are formed via shared group membership (shared workplace, shared school, etc.) or through connections to other friends, though sharing a group or mutual friend over time is not likely to be the reason a friendship ends.

STERGMs were chosen because they are one of the major frameworks competing with SAOMs, and thus, the results would make for an important comparison with the results of the replication using Snijder and colleague's (2010) models. The SAOMs use an actor-based approach, where the focus is on the individual as an agent of change in the network, while the STERGMs use a tie-based approach, which focuses on the probability of a relationship between two actors either forming or dissolving (Krivitsky \& Handcock, 2014).

A third set of models were also created based on Kindermann's (2007) method of identifying peer groups and examining how those groups affect individual outcomes. Kindermann's method uses binomial z tests to evaluate direct, dyadic 
friendships occurring more often than expected by chance to determine group membership. After groups are identified, the number of group members denotes group size and the number of in-group children who remain friends with each child in the group over time can be conceptualized as a measure of network stability (Kindermann, 2007). These measures and others are used to characterize each group and create a "group profile." Then, structural equation modeling (SEM) is used to assess whether the group profile significantly predicts the outcome, when controlling for potential confounds (Kindermann, 2007).

This method differs slightly when analyzing the after-school care data, which do not contain any socio-cognitive map information, such as that which was collected in Kindermann's (2007) study. Creating socio-cognitive maps of social networks involves asking participants to report groups of people in the social network who hang out or spend time together frequently. Researchers use this information to create maps of groups within the network based on consensus between multiple participant reports (Kindermann, 2007). This method does not apply to the current study because the children in the after-school care programs were asked to name their friends in the program, so the friendship nominations were measured directly, rather than having children report on other children's friendships, which would be used to create a sociocognitive map.

Kindermann's analysis method was adapted for use with the after-school care program networks by using SAOMs, rather than SEM. With SAOMs, group member influence on outcomes can be measured directly using a term for average alter effects, explained later. Evidence suggests that SAOM estimates have good convergence with 
those obtained using the Kindermann SEM method, with similar results obtained from both models (Kindermann, DeLay, Laursen, Clark-Shim, \& Yelverton, 2013). The Kindermann method was chosen for use in the current study because it is a method developed for social network analyses with children and it takes a different approach than the other two models because it examines group membership influences, rather than dyad-level influences.

Each model was chosen because it employs a different level of analysis. The SAOMs used to replicate the Gesell and colleagues (2012) analyses focus on the individual level, with individual children acting as drivers of change in the network, since this method assumes that actors in a network are trying to optimize their network position. The STERGMs focus on the dyad level, with the probability of a tie between two actors forming or dissolving as the main focus in modeling. And the third model, based on the work of Kindermann (2007), looks to the group as the source of influence on the individual and the individual as an influence on the group. The models include similar structural and covariate terms, but each provides a different lens through which to view the after-school care networks.

Due to the current exploration of dynamic network models in various disciplines, application to childhood networks is valuable because they are inherently small networks, which are widely studied in the social sciences in other settings. Most models of network change over time were developed to examine large networks and address research questions such as disease path trajectories and computer virus spread. STERGMs and SAOMs are the most popular, well-established longitudinal models 
used in analyses on small social networks, though these were not designed specifically for use with small networks. As such, model estimation may not perform as well for small networks, necessitating the need to use less complex models. More work needs to be done before we can claim to have robust longitudinal social network models for use with small networks in the social sciences. The current study is one small step in continuing that work through the comparison of three competing network models.

\section{Method}

The current study is a new analysis of an existing database. The data were collected by Gesell and colleagues (2012); data collection was approved by the Institutional Review Board at Vanderbilt University (IRB\#090986).

\section{Participants}

Participants in this longitudinal network study were public school children enrolled in one of two separate after-school care programs in an urban area in the southern United States. Both after-school programs ran during the week, Monday through Friday, beginning around 3:00 pm and ending around 6:00 pm. The program held in an elementary school will be referred to as the Coleman program, while the program held in a community center will be referred to as the YMCA program. The Coleman program was initiated at the time the study began, while the YMCA program was already established. Children were excluded from the study if they were less than 5 years old or older than 12 years old. They were also excluded if they had uncontrolled asthma, which would likely affect their activity levels, or if they were not enrolled in school at the time of the study. Eighty-one students total were included in 
the social network analysis portion of the study, with 46 children in the Coleman program and 35 in the YMCA program (Gesell et al., 2012).

The study included a diverse group of children. Of the children in the Coleman program, $48 \%$ identified as African American, 26\% identified as Latino/a, and 26\% identified as White. In the YMCA program, $57 \%$ of the children identified as White, while 29\% identified as African American, $11 \%$ identified as Latino/a, and 3\% identified as Asian. The Coleman program had more female children (59\%) than male children (41\%), as did the $74 \%$ female YMCA program. The average age $(M=7.43$, $S D=1.63$ ) in the YMCA program was less than the average age in the Coleman program $(M=8.39, S D=1.72)$, likely because the oldest children in the YMCA program were 10 years old, while the Coleman program included 11- and 12-yearolds. When age was used as a categorical variable in analyses it was dichotomized into two groups using a median split: older children, aged 9 to 12, and younger children, aged 5 to 8 . See Tables 1.1 and 1.2 for additional demographic and covariate information.

\section{Data Collection}

Study staff collected data during the after-school programs' normal hours. Data were collected at 6-week intervals (three time points total) between February and May 2010 (Gesell et al., 2012). Physical activity was measured with accelerometers (Actigraph) worn by the children for at least 60 minutes per day, while they were at the after-school program, for 5 days, at each time point. Accelerometer data collection is considered highly reliable $(r=0.93)$ and has been used with a wide range of participants. Accelerometer data was used to determine how much time the children 
spent in sedentary, light, moderate, or vigorous activity (Gesell et al., 2012).

Percentage of time spent in moderate and vigorous activity was used as an outcome variable in analyses that incorporated continuous outcomes, but it was converted into deciles for analyses which required categorical outcomes.

Body weight and height were measured during the after-school program and were used to calculate BMI. Children were classified as obese if they were higher than the $95^{\text {th }}$ BMI percentile, overweight if they were between the $85^{\text {th }}$ and $95^{\text {th }}$ percentile, and a healthy weight otherwise. Percentiles were used for classification, rather than raw BMI, due to variations in age and sex of participants, which would influence comparisons in raw BMI scores. A demographic survey was also completed by parents, which included questions on the child's race/ethnicity, sex, which school the child attended, and if the child had siblings in the program, among other questions (Gesell et al., 2012).

To collect social network data, children were interviewed one-on-one by study staff members. The staff asked the children, “"Please tell me the names of the friends you hang around with and talk to and do things with the most here in this after-school program"” (Gesell et al., 2012, p. 1066). The children were asked this at each time point, without being reminded of their previous answers. This method of collecting network data is a nomination-based approach, which has been used since the beginning of network studies. This is one of the most common ways to collect data from small networks, though it does rely on recall and participant interpretation of what it means to be "friends" with someone (Marsden, 2011). These friendship nomination data yielded a directed network of friendship ties. 


\section{Missing Data}

There was no missing network nomination data in either of the after-school care programs. However, eight students from each program (16 children total) were missing attribute data at one or two time points during the study. For each program separately at each time point the amount of missing attribute data ranged from $1 \%$ to $8 \%$, with the total amount of missing attribute data across the three time points of the Coleman program equaling $4 \%$, and the total amount of missing attribute data across the three time points of the YMCA program was $4.5 \%$. Combined, $4.2 \%$ of the attribute data was missing across both programs across all three time points.

Due to the small amount of missing data, the lack of literature about how to handle missing node attribute data in network analyses, and the current inability of STERGMs to employ more advanced missing data methods, such as multiple imputation, mean substitution was used for the missing attribute data. The method used assumes that the data are missing completely at random. There is some evidence suggesting that this method of handling missing data is only slightly biased in $90 \%$ of simulations with as much as $17 \%$ of the data missing completely at random (Schafer \& Graham, 2002). Given that the study accrued very little data loss, we were fortunate that missing data issues were minimal.

\section{Analysis Procedure}

Models were run on each after-school program network separately, as the two networks were dissimilar. The YMCA program was not established as part of the study, but had been running independently prior to the study's commencement. Also, all of the children in the YMCA program attended the same elementary school. The 
Coleman program was created as part of the Gesell et al. (2012) study; it included students from five different schools, as well as a few homeschooled children. The newness of the program and wide variety of schools represented makes it more likely that students would not know each other well at the start of the program, compared to those students who were part of the established YMCA program who all attended the same school together. All analyses were conducted using R version 3.3.2 (2016).

\section{Kindermann Model}

The first models created were based on the work of Kindermann (2007) and were conducted using the Simulation Investigation for Empirical Network Analysis (SIENA) package in R (Ripley, Boitmanis, Snijders, \& Schoenenberger, 2017).

Kindermann's method identifies friendship groups within a network and characterizes each group to create a group profile. Then, structural equation modeling (SEM) is used to assess whether the group profile significantly predicts an outcome, while also including potential confounds in the model (Kindermann, 2007). Using SAOM, group member influence on outcomes can be measured directly. In order to use Kindermann's software to complete these analyses, average group scores would have needed to be calculated for every group a child was connected to, while SAOM handles these steps with two average effect parameters, explained below (Kindermann, personal communication, November 12, 2017).

Evidence suggests that SAOM estimates are similar to those obtained using the Kindermann SEM method. Kindermann and colleagues (2013) utilized both methods and found that peer influence effects were similar across both methods. Odds ratios obtained from the SAOM corresponded well to correlations and betas obtained with 
the Kindermann SEM method. One advantage SAOMs have over the Kindermann method is that they can evaluate whether people in a group become more similar to one another over time, which has not yet been incorporated into the Kindermann SEM method (Kindermann, personal communication, November 12, 2017).

The outcome for these models was activity level, grouped into deciles and the demographic covariate was gender similarity, which is used in many of Kindermann's analyses (Kindermann, 2007; 2013). There was also a term to examine the influence of group size (in-degree). Both average alter and average similarity effects were included in the models. In network analysis, the term "ego" is used to refer to an individual who is the focus of an analysis, while the term "alter" is used to refer to a person who is directly connected to an ego. Each individual node can be conceptualized as an ego and their connections are referred to as their alters. The average alter effect can be thought of as the covariance of a person and their connections' (alters') behaviors (it is their behavior multiplied by the average of their alters' behaviors), while the average similarity effect measures how similar a person (ego) is to their peer group as a whole by taking the average value of the person's connections (alters) and comparing it to that individual's value (Ripley, Snijders, \& Preciado, 2011). In other words, the average similarity effect is how similar a person is to their friend group, while the average alter effect is how a group influences a person's behavior. There was also a term in the models representing transitive triplets, which counts the number of transitive patterns in a network, meaning, when one person $i$ is connected to both person $j$ and person $k$, persons $j$ and $k$ are more like to be connected to each other 
(Ripley, Snijders, \& Preciado, 2011). Linear and quadratic trends were also included in the model.

\section{Separable Temporal Exponential Random Graph Models (STERGMs)}

Two STERGMs were used for each after-school network: a basic STERGM composed only of structural network characteristics and activity level effects, as well

as a full model, which included covariates chosen to match the analyses conducted by Gesell and colleagues (2012). Dissolution models were the same across all analyses they included a term for the main effect of percent of time spent in moderate and vigorous activity based on in-degree (nodeicov), meaning how an individual's friend's level of activity affects the individual's level of activity. Another term was the main effect of percent of time spent in moderate and vigorous activity based on out-degree (nodeocov), meaning how an individual influences their friends' activity levels. The last term was the effect of homophily (nodematch) based on decile of time spent in moderate and vigorous activity, meaning similarity in activity levels between an individual and their friend. Homophily is the tendency to affiliate with similar others and is sometimes referred to as a similarity effect (Handcock, Hunter, Butts, Goodreau, Krivitsky, \& Morris, 2017).

The formation equation for the basic models included the same three terms as the dissolution model, in addition to a term representing the tendency for tie reciprocation (mutual) and a term for popularity based on geometrically weighted outdegree (gwodegree; Handcock et al., 2017). The formation equation for the full models included the previously mentioned reciprocity (mutual), popularity (gwodegree), and activity level homophily (nodematch for activity decile) terms. In 
addition to those terms, there were two more network structure terms included: cyclic triples (ctriple) and transitive triples (ttriple). A transitive triple is the idea that if one person (person $i$ ) is connected to two other people ( $j$ and $k$ ), those two people are more likely to become friends with each other, creating a transitive triple. This is characterized as a tendency toward hierarchy in friendship formation. A cyclic triple has a similar triangle structure: If person $i$ is friends with person $j$ and person $j$ is friends with person $k$, then person $k$ is likely to be friends with person $i$. However, this term is characterized as a non-hierarchical means of friendship formation (Handcock et al., 2017; Krivitsky \& Handcock, 2014). The full models also included additional homophily terms for gender, obesity, race, family member, and age category. The model for the Coleman network included a homophily term for school as well, since children in the Coleman after-school program attended different schools.

Coefficients obtained from the STERGMs are conditional log-odds ratios, where the probability of a friendship tie forming or dissolving based upon a specific parameter is conditional on the other model parameters (Krivitsky \& Handcock, 2014). The log-odds can be converted into odds ratios by taking the exponential of the theta coefficient. A negative parameter value indicates a shorter friendship duration because the model parameters represent friendship persistence (Krivitsky \& Handcock, 2014).

\section{Gesell and Colleagues (2012) Replication}

Lastly, models were created to conduct a replication with extension of the original analyses conducted by Gesell and colleagues (2012) using the SIENA package in R (Ripley et al., 2017). The same terms were included in the models, 
however, the networks were modeled separately, as opposed to the original analysis, where the networks were modeled together as a combined network, with ties between children in the two networks coded as structural zeroes (Gesell et al., 2012). Structural effects included in these models were: out-degree, reciprocity, transitive triplets, and 3-cycles. These are the same as the structural effects included in the STERGMs.

SAOMs also allow the inclusion of dyadic covariate effects, which are represented by a symmetric adjacency matrix where participants have a reciprocal tie if they are matched on the covariate or no tie otherwise (Ripley, Snijders, \& Preciado, 2011). The dyadic covariates included in this analysis were race, living in the same household, and attending the same school (school was included in the Coleman dataset only). Attribute effects were also included for gender, obesity, and age. Three terms were included for all attributes: alter, ego, and similarity. The alter effect is a measure of covariate-related popularity, which is determined by adding the covariate across all of an individuals' alters (Ripley, Snijders, \& Preciado, 2011). The ego effect is defined as a covariate-related activity effect, indicating the individuals' covariate-weighted out-degree (Ripley, Snijders, \& Preciado, 2011, p. 125). The similarity effect is a measure of the similarity of an individual's covariate level to those of their alters' covariate levels, or, how similar a person is to those they are directly connected to in the network (Ripley, Snijders, \& Preciado, 2011). The dependent behavioral variable in this analysis was activity-level, grouped into deciles.

\section{Results}

\section{Kindermann Model}


The Kindermann models fit adequately, with convergence t-ratios between -0.18 and 0.19 , most of which fell between -0.07 and 0.06 . Significance was determined based on $t$-values, which were obtained by dividing the parameter estimate by its standard error. Any $t$-value greater than 1.96 was considered significant at the $\mathrm{p}$ $<0.05$ level. In both the YMCA and Coleman models only the transitive triplets and gender similarity terms were statistically significant. In both networks, children were more likely to become friends if they shared a mutual friend and they were more likely to be friends with children of the same gender.

Results from these models do not provide evidence that children's friend groups are influencing their activity levels. There is also a lack of evidence that group size is important as an influence on a child's friendships, suggesting that children who have larger friendship groups aren't likely to have more friendship ties than children who have smaller friend groups. See Tables 2.1 and 2.2 for full results.

\section{Separable Temporal Exponential Random Graph Models (STERGMs)}

The goodness-of-fit for all STERGMs was sufficient, with Monte Carlo pvalues for model parameters ranging from 0.72 to 1.0 . The closer the p-value is to 1.0 , the better the model fits, as this indicates the difference between the parameters in the observed networks and the simulated networks created based on the model (Handcock et al., 2017). The basic models demonstrated slightly better overall model fit compared to the full models (smaller AIC values), which is likely due to their more parsimonious nature.

For the full model of the YMCA dataset, the mutual, popularity, cyclic triples, obesity homophily, family member homophily, and age category homophily were 
statistically significant for forming friendship ties, while the activity level in-degree term was statistically significant for dropping friendship ties. For the full model for the Coleman dataset, the popularity, transitive triples, cyclic triples, gender homophily, obesity homophily, race homophily, school homophily, family member homophily, and age category homophily were statistically significant for forming friendship ties, while activity level in-degree was statistically significant in dissolving ties.

In the YMCA dataset, a relationship is more likely to occur if it will create a mutual tie; the odds of forming a mutual tie are 2.10 times greater than forming a nonmutual tie. The popularity term indicated there was not a tendency for children to nominate friendships with popular children. There was a significant negative effect of cyclical ties, indicating that triples are not antihierarchical in this network. If a child was obese, he or she was less likely to become friends with other obese children, compared to all other obese and non-obese friendship combinations, with all other covariates and structural characteristics held constant. Children were also less likely to become friends with their own family members and those who were in the same age category (older kids versus younger kids). In terms of dissolution, a relationship was less likely to end if a person's friend's activity level (their alter) is similar to their own, with 5.7 times the odds of continuing the friendship, compared to those whose alters have a dissimilar level of activity. See Table 3.1 for the full results.

The Coleman results also show that children did not have a tendency to nominate friendships with popular children. Friendship triads were not likely to be formed in a transitive nor a cyclical manner. Children were less likely to form friendships with those of the same gender, race, family, age category, or obesity status. 
However, students who went to the same school were more likely to become friends (almost three times the odds), compared to friendships between children from different schools. In terms of dissolution, a relationship was less likely to end if an alter's activity level is similar to their own, with 6.6 times the odds of continuing the friendship, compared to those whose alters have a dissimilar level of activity. See Table 4.1 for the full results.

The basic models included only structural effects and activity level effects. Results from these models for both programs show that reciprocity, popularity, indegree activity level, and out-degree activity level are all significant in forming friendships, while in-degree activity level is also significant in the ending of friendships. Ties were more likely to form if they were reciprocal and children were less likely to endorse being friends with popular children. Children with high levels of activity were less likely to form ties with other children with high levels of activity. But once friendship ties are formed, children have higher odds of continuing the friendship if their alter has a similar activity level.

\section{Gesell and Colleagues (2012) Replication}

The full models fit well, with convergence $t$-ratios between -0.06 and 0.05 . Statistical significance was determined based on $t$-values, which were obtained by dividing the parameter estimate by its standard error. Any $t$-value greater than 1.96 was considered significant at the $p \leq 0.05$ level. Any $t$-value greater than 2.58 was considered significant at the $p \leq 0.01$ level. $T$-values between 1.65 and 1.95 are noted as being at the $p \leq 0.10$ level, though these are only considered approaching statistical significance, as the alpha level was set at 0.05 . Basic models that did not include 
covariate effects were also created for both networks. These models displayed adequate fit with convergence $t$-ratios between -0.16 and 0.07 . The majority of the ratios were between -0.03 and 0.06 .

Significant results from the selection side of the full YMCA model include the structural effects out-degree, reciprocity, transitive triplets, and 3-cycles. Friendship ties were more likely to form if they completed a reciprocal tie or if they completed a transitive triplet (friends of a friend becoming friends). They were less likely to form if the tie completed a 3-cycle. The negative out-degree effect indicates that children were not very likely to have many friendship ties in the network. The dyadic covariate effects of participants being of the same race and living in the same household were also statistically significant. This indicates that children were more likely to form ties with other children of the same race $(\mathrm{OR}=1.25)$, but were less likely to form friendship ties with children living in their household $(\mathrm{OR}=0.19)$. Gender similarity and age similarity were also statistically significant, indicating that children were more likely to become friends with other children of the same gender $(\mathrm{OR}=1.79)$ and of a similar age $(\mathrm{OR}=2.61)$. On the influence side of the model, only the rates of change were statistically significant, indicating that there was more change in friendship ties in the network between time 1 and time 2 than between time 2 and time 3, though both parameters were large. See Table 5.1 for the complete model results.

Selection effects for the full Coleman model that were statistically significant include out-degree, transitive triplets, 3-cycles, the dyadic covariate effect of attending the same school, and the covariate effect of gender similarity. Again, children were not likely to have a large number of friendship ties in the network (out-degree) nor 
were they likely to form ties that would create a 3-cycle. There was a tendency toward transitive triplets in the network. Children were also more likely to become friends with other children who attended the same school as them $(\mathrm{OR}=2.03)$. And they were more likely to become friends with children of the same gender $(\mathrm{OR}=1.54)$. On the influence side of the model, only the rate of change from time 2 to time 3 was statistically significant, indicating that there was more change in friendship ties from time 2 to time 3 than there was from time 1 to time 2. See Table 6.1 for the complete model results.

The basic models for both the YMCA and Coleman networks displayed similar results, with significant rate parameters, significant negative out-degree effects, and significant positive reciprocity effects. However, the Coleman network also had a significant average similarity effect for activity level, indicating that people tended to have a similar level of activity compared to the average activity level of their friends. In other words, the average activity level of an individual's friends influenced their activity level. See Tables 5.2 and 6.2 for full results.

\section{Discussion}

In our study we learned that when using STERGMs, a modeling technique that focuses on the probability of a pair of actors forming or dissolving a tie, activity levels may not influence the formation of friendship ties, but they may play a part in friendships ending. In both the Coleman and YMCA programs, a relationship was less likely to end if a person's friend's activity level (their alter) is similar to their own, compared to those whose alters had a dissimilar level of activity. 
We also learned that characteristics of children in the two networks had different levels of influence on friendship formation, with shared school as a key component of friendships in the Coleman program. On the other hand, children in the YMCA network were more likely to be friends with children who shared their race/ethnicity. Children in both networks displayed a tendency to become friends with those who were of a similar age or the same gender as themselves, though this tendency did not hold across all models. Obesity did not seem to be very influential in friendship formation in either network.

The lack of evidence that activity level is influential in children's relationships is not entirely surprising. As can be seen in Figures 1 and 2, children with high activity levels and children with low activity levels were not grouped together exclusively in the networks at any time point. Instead, activity level seems to be randomly dispersed throughout the networks. At the first time point in the Coleman network, the children who have the highest activity levels also appear to have the most friends, however, at the second time point the children with the most friends tend to be toward the middle of the activity level spectrum; one child with a low activity level and another child with a high activity level also had a large number of friends. At the final time point, children with the lowest and highest activity levels seem to be grouped together on one side of the network, while children with intermediate activity levels seem to be grouped together on the other side of the network. In the YMCA network, children with the lowest activity levels had the most friends at time 1 and time 2, while the children who have the most friends at time three display a wide range of activity levels. 
Finally, we were able to compare the results of three models, each of which had a different way of examining the networks. The Kindermann model included influences between an individual and their peer group, while the STERGMs modeled the probability of children forming and dissolving ties based on behavior and a set of covariates. The SAOMs included influence and selection effects, which parse out the difference between forming a friendship due to shared characteristics or behaviors and becoming more like another person (i.e., having similar activity levels) due to friendship. While a few of the findings were convergent across all models, not all of the findings were the same.

\section{Kindermann Model}

The Kindermann model did not produce many significant results with these data, but the results that were significant are informative because they support the literature on gender similarity in children's relationships and the network literature in sociology. The significance of gender similarity suggests that children were more likely to become friends with other children of the same gender, compared to children of the opposite gender, in both after-school care programs. This provides further evidence to support the literature that children tend to form same-sex friendships, rather than opposite-sex friendships (e.g., Eccles, Wigfield, \& Schiefele, 1998;

Kindermann, 2007). The significance of the transitive triplets term in both the YMCA and Coleman models suggests that friends of a friend are likely to become friends with one another, supporting the network sociology literature, which suggests that transitive triplets are common in networks (e.g., Wasserman \& Faust, 1994).

\section{Separable Temporal Exponential Random Graph Models (STERGMs)}


The outcome of interest was activity level; activity level effects were not statistically significant in the formation sections of the full STERGMs, which also included additional covariate effects. Instead, the full models for both networks show that while having the same level of activity as another child may not influence a child's choice to become friends with someone else, having the same level of activity as an already existing friend is likely to continue the friendship, compared to having a dissimilar activity level.

Results from the full model for the YMCA program show that children were less likely to become friends with their family members and those who were in the same age group as themselves. Obese children were also less likely to become friends with other obese children. The full model for the Coleman program had the same covariate results, in addition to some differences: Children were less likely to become friends with other children of the same race/ethnicity or sex, and were more likely to become friends with children who attended the same school. These differences between covariate effects in friendship formation between the programs suggest that the various factors that contribute to children becoming friends with one another are not only meaningful but may vary significantly across different networks.

\section{Gesell and Colleagues (2012) Replication}

Results from the replication models showed that the two networks, Coleman and YMCA, are different in terms of selection effects, which provides support for the idea that there is significant and meaningful variation in factors affecting friendship formation across different social networks. In the Coleman program, children were more likely to become friends with other children if they attended the same school, 
whereas, in the YMCA program, children were more likely to become friends with other children of the same race/ethnicity. Children in the YMCA program were also less likely to be friends with other children in their family, while this parameter was not significant in the Coleman program. Both programs showed a tendency for children to become friends with other children of the same gender, while children in the YMCA program also tended to become friends with other children based on similarity in age. The replication results also suggest that friends' activity level did not significantly influence a child's level of activity.

\section{Kindermann, STERGM, and Gesell and Colleagues (2012) Replication \\ Comparison}

One term that was statistically significant across the STERGMs and the replication SAOM models of the Coleman program was school. People were more

likely to be friends if they went to the same school. This is important because it points to the idea of familiarity - children in the Coleman program chose to be friends with other children from the same school, which may be attributed to the fact that the Coleman program was established when the study began, so the children who went to different schools may not have known one another.

There were various discrepancies between results of the three models, however. SAOM results show that children in the YMCA program were more likely to be friends with children of the same gender and a similar age, which was also supported by findings from the Kindermann model, whereas the STERGM results suggested that this was not the case. The SAOM results for the Coleman program show that children were more likely to be friends with those of the same gender, 
which is also contradictory to the findings in the STERGM model but was supported by the Kindermann model results. While these discrepancies may be due to the difference in underlying model assumptions and varying degrees of model complexity, is it intriguing that the differences were so stark.

Due to the differences between these models, it is recommended that researchers choose one, either STERGM, SAOM, or Kindermann. Researchers should choose a method based on how they think the tie-formation process occurs in a network grounded in theory on the social processes assumed to underlie the network changes. Does tie formation depend on an individual comparing others whom they could form a tie with in the network? In other words, does the individual have agency within a network to decide with whom they will be connected? If the individual weighs potential connections to others and chooses the most rewarding ties an SAOM would be suitable. If the individual looks at each potential tie in isolation to determine whether or not it will be rewarding, a STERGM would be most suitable. This reflects the underlying notion of multinomial (SAOM) versus binary (STERGM) decision making (Block, Stadtfeld, \& Snijders, 2017).

For example, choosing to be friends with one person means you are less likely to become friends with another person because you would be investing time in the first person you chose. On the other hand, choosing to be Facebook friends with someone does not mean you are less likely to become Facebook friends with other people, because you are probably not investing a lot of time in your Facebook friends. Block and colleagues' (2017) "rule of thumb" is to decide whether or not an individual in the network can have unlimited ties in the network or not. If the amount is limited (e.g., 
having a group of close friends), chose an SAOM. If it is unlimited (e.g., Facebook friends or Twitter followers), chose a STERGM.

Another consideration is this: is the social process actor-oriented or tieoriented? Block and colleagues (2017) provide the example of a network of flight connections between airports; a STERGM is more appropriate here because the focus is on the ties, i.e., flights, rather than the airports themselves (no airport is more important because it is the destination, as opposed to being the origin of the flight). However, if the theory is more about the individual and how they are influencing their network (e.g., having individual students on a college campus learn about healthy eating and then educate their peers about healthy food choices), an SAOM is a better choice.

\section{Limitations}

\section{Original Study}

There is some criticism regarding nomination-based network data collection, particularly when working with children, suggesting that it may actually increase bias. For example, Leung (1996) found that Chinese children tended to be biased when asked to report their friendships with other children, compared to peer observations of their friendship groups. The children endorsed more friendships with peers they considered "popular" or in other ways socially desirable, while underreporting friendships with undesirable peers in the nomination-based data collection. Luckily, the tendency to endorse friendships with popular peers can be evaluated in network models to see whether or not this bias is occurring. Results from both the STERGM 
and SAOM replication models suggest that children were not endorsing many friendships with popular peers, so they likely were not exhibiting this bias.

Another type of bias that could be induced by interviewing children is recall bias: Can the children accurately remember who their friends are in the after-school care program? Research suggests that older children are less prone to recall bias as they have more highly developed cognitive skills involving memory (Greenhoot, 2011). Studies have generally found that children tend to inaccurately report their past experiences and, as this is different between age groups, the older group in this study may have reported their friendships more accurately, while the younger children may have been more prone to errors.

Gesell and colleagues (2012) suggest that after-school care networks may differ from other networks of children, particularly with regard to reciprocity, which they found to be lower, compared to other child friendship networks. However, there was still a significant reciprocity effect in the YMCA programs, suggesting that children are more likely to endorse a reciprocal tie than not endorse it, particularly in a program that is well-established, like the YMCA program.

\section{Current Study}

One limitation in this study was sample size. The Coleman program only had 46 children, while the YMCA program had 35 children. This limitation could have impacted the models by contributing to a lack of power to detect significant differences, particularly for model terms that have small effect sizes. Though sample size was small, the networks were not sparse; there were many friendship connections between children in each network. Kolaczyk and Krivitsky (2015) suggest that 
effective sample size in network models is sensitive to sparseness (a lack of ties) and the models being applied to the networks. For example, some model terms are more complicated than others, and thus, the effective sample size varies depending on model complexity (Kolaczyk \& Kritisky, 2015). This was an issue when applying STERGMs to the Coleman and YMCA networks. STERGMs with the same parameters as the SAOMs would not converge, likely due to small sample size and model complexity. Thus, the STERGMs were simplified slightly to allow model convergence; they included only similarity effects of covariates, rather than similarity, ego, and alter effects of covariates.

A limitation in SAOM is that the dependent variable, in this case, the children's activity levels, must be a categorical variable. Activity level was calculated as a continuous variable representing the percentage of time children spent in moderate or vigorous activity. For the Kindermann and replication analyses, which were conducted using SIENA, activity level was split into deciles. This may not be the best representation of the data. For example, the children at the higher end of the second decile may be more like the children at the lower end of the third decile, compared to children at the lower end of the second decile, yet they are grouped with those other children.

Another limitation is that the STERGMs and replication models were not exactly alike. As previously mentioned, the STERGMs needed to be simplified in order for the models to converge. The STERGMs did not include covariate ego and alter effects, nor did they include rate and shape parameters. This simplification could 
have influenced the results and comparisons between the models because the STERGMs were more parsimonious than the SAOMs.

\section{Future Directions}

One way to investigate these findings further would be to include more afterschool care programs in analyses to see if results are similar or different between other programs. While there were similarities between the Coleman and YMCA programs, there were also some important differences and collecting more data could help shed light on whether other programs share similarities with these two programs or whether there are even more between-program differences that should be explored. The analyses could also benefit from additional sample size - collecting data on larger networks of children could improve overall model fit and parameter estimation. Additional sample size would also allow for the inclusion of more covariates in analyses to see if there are other constructs influencing the relationship between individual activity level and peer influences.

Another path forward would be to conduct similar analyses with different health behavior outcomes, such as eating healthy foods, wearing sunscreen, or decreasing stress. Intervening in after-school care programs could provide an opportunity to promote any of these healthy behaviors. The same or similar covariates could be included in these analyses, as they are important for how children become friends with one another. Obesity could be replaced with a measure of stress or frequency of sunscreen use, while gender, age, race/ethnicity, shared household, and shared school could be retained in the analyses.

\section{Conclusion}


The use of STERGMs and SAOMs in this study allowed us to explore relationship-based influences between children at the level of the individual, the dyad, and the group. The findings suggest that activity level may not be important when children are forming friendships. This, combined with the finding that children are less likely to end a friendship with someone who has a similar activity level to their own, suggests that the most useful interventions to increase activity levels among children may target pairs of children who are already friends or pre-existing groups of friends. Rather than intervening with a few children and hoping that they will influence the behavior of others in the network, intervening in friendship pairs and groups so the children can increase their activity levels together may provide the most benefit. 


\section{References}

Anderson, A., Locke, J., Kretzmann, M., \& Kasari, C. (2016). Social network analysis of children with autism spectrum disorder: Predictors of fragmentation and connectivity in elementary school classrooms. Autism, 20(6), 700-709.

Block, P., Stadtfeld, C., \& Snijders, T. A. (2017). Forms of Dependence: Comparing SAOMs and ERGMs from Basic Principles. Sociological Methods \& Research, 1-57.

Cooc, N., \& Kim, J. S. (2017). Peer influence on children's reading skills: A social network analysis of elementary school classrooms. Journal of Educational Psychology, 109(5), 727-761.

DeLay, D., Ha, T., Van Ryzin, M., Winter, C., \& Dishion, T. J. (2016). Changing friend selection in middle school: A social network analysis of a randomized intervention study designed to prevent adolescent problem behavior. Prevention Science, 17(3), 285-294.

Eccles, J. S., Wigfield, A., \& Schiefele, U. (1998). Motivation to succeed. In W. Damon \& N. Eisenberg (Eds.), Handbook of Child Psychology: Volume 3. Social, Emotional, and Personality Development (5 ${ }^{\text {th }}$ ed., p. 1017-1095). New York: Wiley.

Feiring, C., \& Lewis, M. (1989). The social networks of girls and boys from early through middle childhood. In D. Belle (Ed.), Children's Social Networks and Social Supports, 119-150.

Gesell, S. B., Tesdahl, E., \& Ruchman, E. (2012). The distribution of physical activity in an after-school friendship network. Pediatrics, 129, 1064-71. 
Golemiec, M., Schneider, J., Boyce, W. T., Bush, N. R., Adler, N., \& Levine, J. D. (2016). Layered social network analysis reveals complex relationships in kindergarteners. Frontiers in Psychology, 7, 276-282.

Greenhoot, A. F. (2011). Retrospective methods in developmental science. In B. Larsen, T. D., Little, \& N. A. Card (Eds.) Handbook of developmental research methods, 1, 196-210.

Handcock, M., Hunter, D., Butts, C., Goodreau, S., Krivitsky, P., \& Morris, M. (2017). ergm: Fit, simulate and diagnose exponential-family models for networks, The Statnet Project (<URL: http://www.statnet.org>). R package version 3.6.1.

Kindermann, T. A. (2007). Effects of naturally existing peer groups on changes in academic engagement in a cohort of sixth graders. Child Development, 78(4), 1186-1203.

Kindermann, T. A., DeLay, D., Laursen, B., Clark-Shim, H., \& Yelverton, R. (2013) Convergent findings about peer group influences on classroom engagement in a small town from analyses using RSiena and SEM. Poster presented at the biennial meetings of the Society for Research in Child Development. Seattle, WA.

Kolaczyk, E. D., \& Csárdi, G. (2014). Statistical analysis of network data with R. New York: Springer.

Kolaczyk, E. D., \& Krivitsky, P. N., (2015). On the question of effective sample size in network modelling: An asymptotic inquiry, Statistical Science: A Review Journal of the Institute of Mathematical Statistics, 30(2), 184-198. 
Krivitsky, P. N., \& Handcock, M. S. (2014). A separable model for dynamic networks. Journal of the Royal Statistical Society: Series B (Statistical Methodology), 76(1), 29-46.

Krivitsky, P., \& Handcock, M. (2016). Stergm: Fit, simulate, and diagnose models for network evolution base on exponential-family random graph models, The Statnet Project (<URL: http://www.statnet.org>). R package version 3.4.0.

Laninga-Wijnen, L., Ryan, A. M., Harakeh, Z., Shin, H., \& Vollebergh, W. A. (2017). The moderating role of popular peers' achievement goals in 5th-and 6thgraders' achievement-related friendships: A social network analysis. Journal of Educational Psychology, 1-19.

Leung, M-C. (1996). Social networks and self enhancement in Chinese children: A comparison of self reports and peer reports of group membership. Social Development, 5(2), 146-157.

Marsden, P. V. (2011). Survey methods for network data. In P. Carrington \& J. Scott (Eds.) The SAGE Handbook of Social Network Analysis, (p. 370-388). Thousand Oaks, CA: SAGE.

R Core Team (2016). R: A language and environment for statistical computing. $R$ Foundation for Statistical Computing, Vienna, Austria. URL https://www.Rproject.org/.

Ripley, R., Boitmanis, K., Snijders, T. A. B., \& Schoenenberger, F. (2017). RSiena: Siena - simulation investigation for empirical network analysis. R package version 1.2-3. URL <https://CRAN.R-project.org/package=RSiena>. 
Ripley, R. M., Snijders, T. A., \& Preciado, P. (2011). Manual for RSIENA. University of Oxford, Department of Statistics, Nuffield College, 1-234.

Schafer, J. L., \& Graham, J. W. (2002). Missing data: Our view of the state of the art. Psychological Methods, 7(2), 147-177.

Snijders, T. A. B., van de Bunt, G. G., \& Steglich, C. E. G. (2010). Introduction to stochastic actor-based models for network dynamics. Social Networks, 32, 4460.

Valente, T. W., Fujimoto, K., Chou, C. P., \& Spruijt-Metz, D. (2009). Adolescent affiliations and adiposity: A social network analysis of friendships and obesity. The Journal of Adolescent Health, 45(2), 202-204.

Wasserman, S., \& Faust, K. (1994). Social network analysis: Methods and applications. Cambridge, UK: Cambridge University Press. 


\section{APPENDICES}

Table 1.1 YMCA demographics

\begin{tabular}{llc} 
Variable & Levels & \% \\
\hline Gender & Female & 74 \\
& Male & 26 \\
\hline Race & Asian & 3 \\
& Black & 29 \\
& Hispanic & 11 \\
& White & 57 \\
\hline Family $^{\dagger}$ & No & 69 \\
& Yes & 31 \\
\hline Age category & Older & 40 \\
& Younger & 60 \\
\hline Obese & No & 83 \\
& Yes & 17
\end{tabular}

${ }^{\dagger}$ Family denotes whether or not a child has at least one family member in the network

*Age category: 9-12 year-olds are in the older group, 5-8 year-olds are in the younger group 
Table 1.2 Coleman demographics

\begin{tabular}{llc} 
Variable & Levels & \% \\
\hline Gender & Female & 59 \\
& Male & 41 \\
\hline Race & Black & 48 \\
& Hispanic & 26 \\
& White & 26 \\
\hline Family ${ }^{\dagger}$ & No & 48 \\
& Yes & 52 \\
\hline Age category & Older & 57 \\
& Younger & 43 \\
\hline Obese & No & 76 \\
& Yes & 24 \\
\hline School & Homeschool & 7 \\
& School 1 & 2 \\
& School 2 & 33 \\
& School 3 & 2 \\
& School 4 & 45 \\
& School 5 & 11 \\
\hline
\end{tabular}

'Family denotes whether or not a child has at least one family member in the network

¥Age category: 9-12 year-olds are in the older group, 5-8 year-olds are in the younger group 
Table 2.1 YMCA results using Kindermann model

\begin{tabular}{lccc} 
Model term & Estimate & SE & Odds ratio $(95 \%$ CI) \\
\hline Ties: Linear tendency & 0.12 & 0.11 & $1.13(0.91,1.40)$ \\
Ties: Quadratic tendency & -0.09 & 0.19 & $0.91(0.63,1.32)$ \\
Ties: Transitive triplets & $0.18^{*}$ & 0.03 & $1.20(1.13,1.27)$ \\
Group influence on activity level & 0.29 & 0.48 & $1.34(0.52,3.43)$ \\
Activity level similarity (person-to-group) & -9.37 & 16.85 & $0.00009\left(4.09 \mathrm{e}^{-19}\right.$ \\
& & & $\left.1.98 \mathrm{e}^{10}\right)$ \\
Group size (in degree) & -0.02 & 0.02 & $0.98(0.94,1.02)$ \\
Gender similarity & $0.35^{*}$ & 0.11 & $1.42(1.14,1.76)$ \\
${ }^{* p}<0.05$ & & &
\end{tabular}


Table 2.2 Coleman results using Kindermann model

\begin{tabular}{lccc} 
Model term & Estimate & SE & Odds ratio $(\mathbf{9 5 \%} \mathbf{C I})$ \\
\hline Ties: Linear tendency & 0.04 & 0.11 & $1.04(0.84,1.29)$ \\
Ties: Quadratic tendency & 0.03 & 0.07 & $1.03(0.90,1.18)$ \\
Ties: Transitive triplets & $0.44^{*}$ & 0.07 & $1.55(1.35,1.78)$ \\
Group influence on activity level & 0.11 & 0.15 & $1.12(0.83,1.50)$ \\
Activity level similarity (person-to-group) & 3.22 & 6.67 & $25.03(0.00005$, \\
& & & $11,914,600)$ \\
Group size (in degree) & -0.02 & 0.02 & $0.98(0.94,1.02)$ \\
Gender similarity & $0.42^{*}$ & 0.12 & $1.52(1.20,1.92)$ \\
$* \mathrm{p}<0.05$ & & &
\end{tabular}


Table 3.1 YMCA results from STERGM full model

\begin{tabular}{lcccc} 
Model term & Estimate & Std. Error & p-value & Odds ratio (95\% CI) \\
\hline $\begin{array}{l}\text { Formation } \\
\text { mutual }\end{array}$ & 0.742 & 0.176 & $<0.0001^{*}$ & $2.10(1.49,2.97)$ \\
gwodegree & -9.369 & 1.092 & $<0.0001^{*}$ & $0.00008(0.000009$, \\
& & & & $0.0007)$ \\
ttriple & -0.002 & 0.016 & 0.874 & $0.998(0.97,1.03)$ \\
ctriple & -0.167 & 0.044 & $0.0002^{*}$ & $0.85(0.78,0.93)$ \\
nodematch.gender & -0.035 & 0.130 & 0.786 & $0.97(0.75,1.25)$ \\
nodematch.obese & -0.723 & 0.113 & $<0.0001^{*}$ & $0.49(0.39,0.61)$ \\
nodematch.race & -0.195 & 0.128 & 0.128 & $0.82(0.64,1.05)$ \\
nodematch.family & -0.695 & 0.117 & $<0.0001 *$ & $0.50(0.40,0.63)$ \\
nodematch.mod_vig & -0.230 & 0.249 & 0.356 & $0.79(0.48,1.29)$ \\
nodematch.age & -0.395 & 0.124 & $0.001 *$ & $0.67(0.53,0.85)$ \\
& & & & \\
Dissolution & & & & \\
nodeicov.mod_vig & 1.740 & 0.787 & $0.028 *$ & $5.70(1.22,26.66)$ \\
nodeocov.mod_vig & 0.422 & 0.788 & 0.593 & $1.53(0.33,7.17)$ \\
nodematch.mod_vig & 0.285 & 0.357 & 0.425 & $1.33(0.66,2.68)$
\end{tabular}


Table 3.2 YMCA results from STERGM basic model

\begin{tabular}{lcccc} 
Model term & Estimate & Std. Error & p-value & Odds ratio (95\% CI) \\
\hline $\begin{array}{l}\text { Formation } \\
\text { mutual }\end{array}$ & 0.482 & 0.152 & $0.0016^{*}$ & $1.62(1.20,2.18)$ \\
gwodegree & -8.087 & 1.014 & $<0.0001 *$ & $0.0003(0.00004$, \\
& & & & $0.002)$ \\
nodeicov.mod_vig & -6.671 & 0.530 & $<0.0001 *$ & $0.001(0.0004,0.003)$ \\
nodeocov.mod_vig & -2.237 & 0.433 & $<0.0001 *$ & $0.11(0.05,0.26)$ \\
nodematch.mod_vig & -0.266 & 0.250 & 0.2870 & $0.77(0.47,1.26)$ \\
& & & & \\
Dissolution & & & & \\
$\quad$ nodeicov.mod_vig & 1.740 & 0.787 & $0.028 *$ & $5.70(1.22,26.66)$ \\
$\quad$ nodeocov.mod_vig & 0.422 & 0.788 & 0.593 & $1.53(0.33,7.17)$ \\
nodematch.mod_vig & 0.285 & 0.357 & 0.425 & $1.33(0.66,2.68)$
\end{tabular}


Table 4.1 Coleman results from STERGM full model

\begin{tabular}{lcccc} 
Model term & Estimate & Std. Error & p-value & Odds ratio (95\% CI) \\
\hline $\begin{array}{l}\text { Formation } \\
\text { mutual }\end{array}$ & 0.252 & 0.177 & 0.154 & $1.29(0.91,1.82)$ \\
gwodegree & -3.517 & 0.364 & $<0.0001^{*}$ & $0.03(0.01,0.06)$ \\
$\quad$ ttriple & -0.097 & 0.027 & $0.0004^{*}$ & $0.91(0.86,0.96)$ \\
ctriple & -0.273 & 0.072 & $0.0002^{*}$ & $0.76(0.66,0.88)$ \\
nodematch.gender & -0.562 & 0.119 & $<0.0001^{*}$ & $0.57(0.45,0.72)$ \\
nodematch.obese & -1.268 & 0.112 & $<0.0001^{*}$ & $0.28(0.22,0.35)$ \\
nodematch.race & -0.650 & 0.132 & $<0.0001^{*}$ & $0.52(0.40,0.67)$ \\
nodematch.school & 1.094 & 0.149 & $<0.0001^{*}$ & $2.99(2.23,4.00)$ \\
nodematch.family & -0.808 & 0.125 & $<0.0001^{*}$ & $0.45(0.35,0.57)$ \\
nodematch.mod_vig & -0.348 & 0.250 & 0.164 & $0.71(0.43,1.16)$ \\
nodematch.age & -0.683 & 0.119 & $<0.0001^{*}$ & $0.51(0.40,0.64)$ \\
& & & & \\
Dissolution & & & & \\
nodeicov.mod_vig & 1.895 & 0.794 & $0.018 *$ & $6.65(1.40,31.53)$ \\
nodeocov.mod_vig & 1.244 & 0.808 & 0.125 & $3.47(0.71,16.91)$ \\
$\quad$ nodematch.mod_vig & 1.107 & 0.623 & 0.076 & $3.03(0.89,10.27)$
\end{tabular}


Table 4.2 Coleman results from STERGM basic model

\begin{tabular}{lcccc} 
Model term & Estimate & Std. Error & p-value & Odds ratio (95\% CI) \\
\hline $\begin{array}{l}\text { Formation } \\
\text { mutual }\end{array}$ & 1.107 & 0.178 & $<0.0001^{*}$ & $3.03(2.14,4.29)$ \\
gwodegree & -0.996 & 0.463 & $0.032^{*}$ & $0.37(0.15,0.91)$ \\
nodeicov_mod_vig & -3.371 & 0.364 & $<0.0001^{*}$ & $0.03(0.01,0.06)$ \\
nodeocov.mod_vig & -4.603 & 0.419 & $<0.0001^{*}$ & $0.01(0.004,0.02)$ \\
nodematch.mod_vig & -0.326 & 0.247 & 0.187 & $0.72(0.44,1.17)$ \\
& & & & \\
Dissolution & & & & \\
$\quad$ nodeicov.mod_vig & 1.895 & 0.794 & $0.018^{*}$ & $6.65(1.40,31.53)$ \\
$\quad$ nodeocov.mod_vig & 1.244 & 0.808 & 0.125 & $3.47(0.71,16.91)$ \\
$\quad$ nodematch.mod_vig & 1.107 & 0.623 & 0.076 & $3.03(0.89,10.27)$
\end{tabular}


Table 5.1 YMCA results from Gesell replication - full model

Estimate (SE)

Odds ratio $(95 \% \mathrm{CI})$

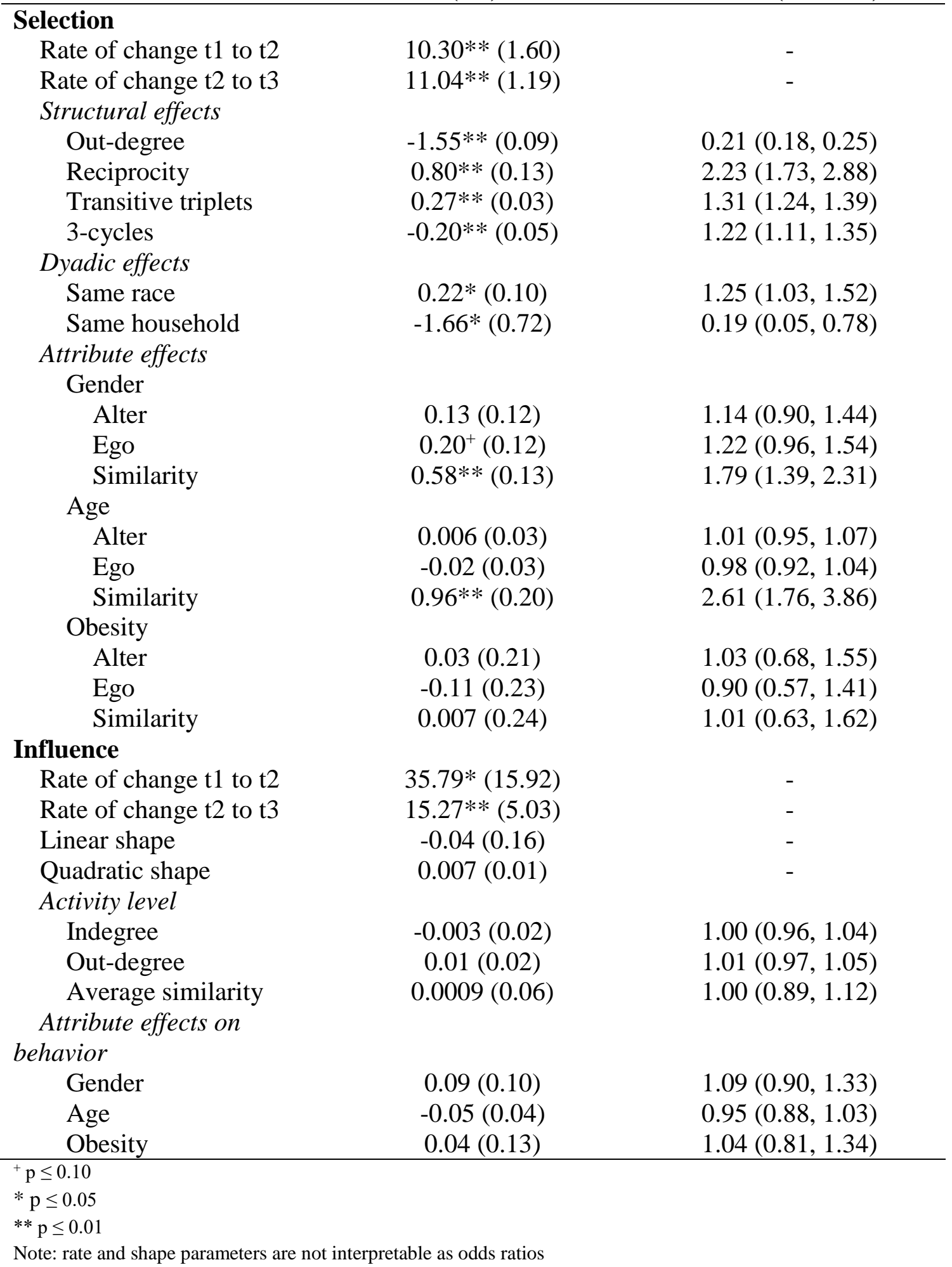


Table 5.2 YMCA results from Gesell replication - basic model

Estimate (SE)

Odds ratio $(95 \% \mathrm{CI})$

\begin{tabular}{|c|c|c|}
\hline Selection & & \\
\hline Rate of change $t 1$ to $t 2$ & $8.80 * *(0.91)$ & - \\
\hline Rate of change $t 2$ to $t 3$ & $9.53 * *(0.96)$ & - \\
\hline Structural effects & & \\
\hline Out-degree & $-0.93 * *(0.06)$ & $0.39(0.35,0.44)$ \\
\hline Reciprocity & $0.96 * *(0.13)$ & $2.61(2.02,3.37)$ \\
\hline Influence & & \\
\hline Rate of change $t 1$ to $t 2$ & $35.08 *(15.18)$ & - \\
\hline Rate of change $t 2$ to $t 3$ & $15.59 * *(3.92)$ & - \\
\hline Linear shape & $0.04(0.16)$ & - \\
\hline Quadratic shape & $0.009(0.01)$ & - \\
\hline Activity level & & \\
\hline Indegree & $-0.03(0.02)$ & $0.97(0.93,1.01)$ \\
\hline Out-degree & $0.02(0.02)$ & $1.02(0.98,1.06)$ \\
\hline Average similarity & $0.03(0.06)$ & $1.03(0.92,1.16)$ \\
\hline${ }^{+} p \leq 0.10$ & & \\
\hline$* \mathrm{p} \leq 0.05$ & & \\
\hline$* * \mathrm{p} \leq 0.01$ & & \\
\hline
\end{tabular}


Table 6.1 Coleman results from Gesell replication - full model

\begin{tabular}{|c|c|c|}
\hline \multicolumn{3}{|l|}{ Selection } \\
\hline Rate of change $t 1$ to $t 2$ & $8.49 * *(1.23)$ & - \\
\hline Rate of change $t 2$ to $t 3$ & $1.93 * *(0.23)$ & - \\
\hline \multicolumn{3}{|l|}{ Structural effects } \\
\hline Out-degree & $-1.59 * *(0.11)$ & $0.20(0.16,0.25)$ \\
\hline Reciprocity & $0.46^{+}(0.26)$ & $1.58(0.95,2.63)$ \\
\hline Transitive triplets & $0.37 * *(0.05)$ & $1.45(1.31,1.60)$ \\
\hline 3-cycles & $-0.16^{*}(0.08)$ & $0.85(0.73,0.99)$ \\
\hline \multicolumn{3}{|l|}{ Dyadic effects } \\
\hline Same race & $0.19(0.13)$ & $1.21(0.94,1.56)$ \\
\hline Same household & $0.46(0.37)$ & $1.58(0.77,3.26)$ \\
\hline Same school & $0.71 * *(0.14)$ & $2.03(1.54,2.67)$ \\
\hline \multicolumn{3}{|l|}{ Attribute effects } \\
\hline \multicolumn{3}{|l|}{ Gender } \\
\hline Alter & $0.05(0.16)$ & $1.05(0.77,1.44)$ \\
\hline Ego & $-0.10(0.17)$ & $0.90(0.64,1.26)$ \\
\hline Similarity & $0.43 * *(0.13)$ & $1.54(1.19,1.99)$ \\
\hline \multicolumn{3}{|l|}{ Age } \\
\hline Alter & $0.03(0.04)$ & $1.03(0.95,1.11)$ \\
\hline Ego & $-0.005(0.05)$ & $1.00(0.91,1.10)$ \\
\hline Similarity & $0.46(0.29)$ & $1.58(0.89,2.79)$ \\
\hline \multicolumn{3}{|l|}{ Obesity } \\
\hline Alter & $-0.04(0.16)$ & $0.96(0.70,1.31)$ \\
\hline Ego & $0.12(0.19)$ & $1.13(0.78,1.64)$ \\
\hline Similarity & $0.10(0.18)$ & $1.11(0.78,1.58)$ \\
\hline \multicolumn{3}{|l|}{ Influence } \\
\hline Rate of change $\mathrm{t} 1$ to $\mathrm{t} 2$ & $19.68^{+}(11.63)$ & - \\
\hline Rate of change $t 2$ to $t 3$ & $9.78 * *(3.78)$ & - \\
\hline Linear shape & $-0.07(0.14)$ & - \\
\hline Quadratic shape & $-0.01(0.02)$ & - \\
\hline \multicolumn{3}{|l|}{ Activity level } \\
\hline Indegree & $-0.03(0.02)$ & $0.97(0.93,1.01)$ \\
\hline Out-degree & $0.04(0.03)$ & $1.04(0.98,1.10)$ \\
\hline Average similarity & $0.17(0.11)$ & $1.19(0.96,1.48)$ \\
\hline \multicolumn{3}{|l|}{ Attribute effects on } \\
\hline \multicolumn{3}{|l|}{ behavior } \\
\hline Gender & $0.24^{+}(0.13)$ & $1.27(0.98,1.64)$ \\
\hline Age & $-0.004(0.03)$ & $1.00(0.94,1.06)$ \\
\hline Obesity & $0.09(0.13)$ & $1.09(0.84,1.41)$ \\
\hline
\end{tabular}


Table 6.2 Coleman results from Gesell replication - basic model

Estimate (SE)

\begin{tabular}{|c|c|c|}
\hline \multicolumn{3}{|l|}{ Selection } \\
\hline Rate of change $t 1$ to $t 2$ & $6.75^{* *}(0.69)$ & - \\
\hline Rate of change $t 2$ to $t 3$ & $1.90 * *(0.23)$ & - \\
\hline \multicolumn{3}{|l|}{ Structural effects } \\
\hline Out-degree & $-1.01 * *(0.07)$ & $0.36(0.31,0.41)$ \\
\hline Reciprocity & $1.08 * *(0.15)$ & $2.94(2.19,3.94)$ \\
\hline \multicolumn{3}{|l|}{ Influence } \\
\hline Rate of change $t 1$ to $t 2$ & $20.26 * *(5.66)$ & - \\
\hline Rate of change $t 2$ to $t 3$ & $10.32 * *(2.15)$ & - \\
\hline Linear shape & $-0.02(0.12)$ & - \\
\hline Quadratic shape & $-0.003(0.01)$ & - \\
\hline \multicolumn{3}{|l|}{ Activity level } \\
\hline Indegree & $-0.03(0.02)$ & $0.97(0.93,1.01)$ \\
\hline Out-degree & $0.02(0.03)$ & $1.02(0.96,1.08)$ \\
\hline Average similarity & $0.18 *(0.07)$ & $1.20(1.05,1.38)$ \\
\hline \multicolumn{3}{|l|}{${ }^{+} p \leq 0.10$} \\
\hline$* \mathrm{p} \leq 0.05$ & & \\
\hline$* * \mathrm{p} \leq 0.01$ & & \\
\hline
\end{tabular}


Figure 1. Change in Coleman network over time. Node size represents degree (how many friends a child has), while color represents activity level (dark purple is lowest, gray in the middle, dark green is highest)

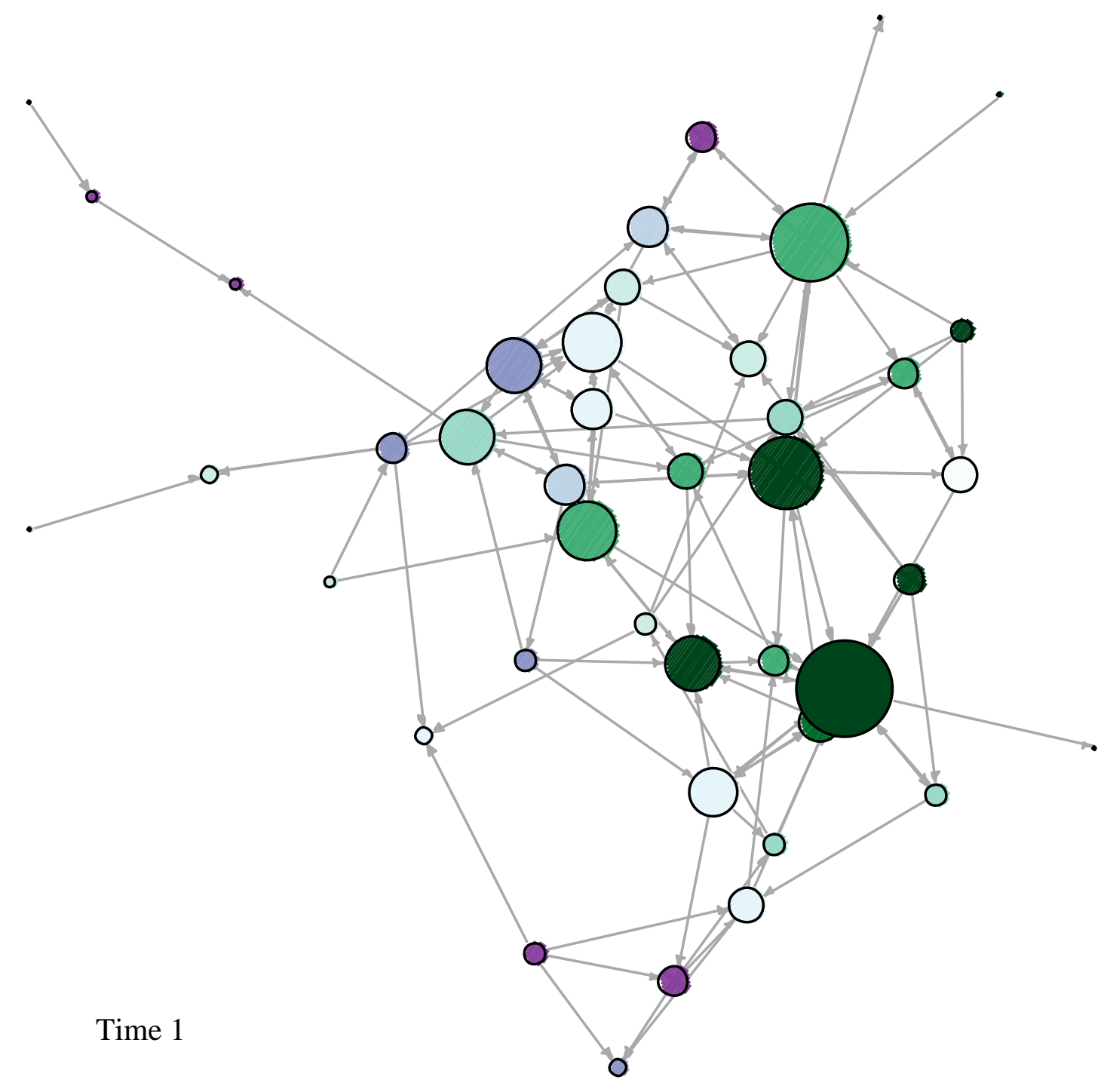




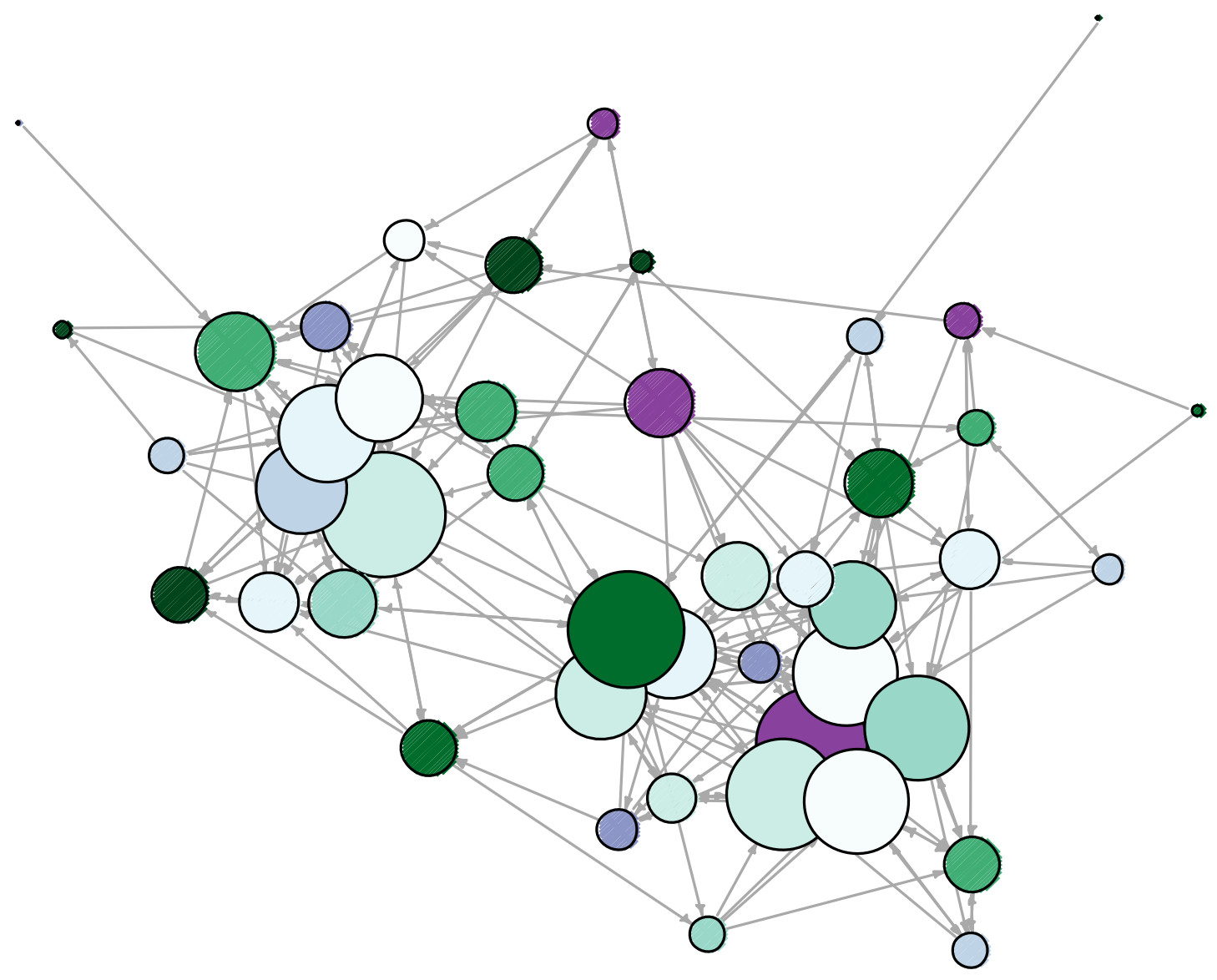

Time 2 


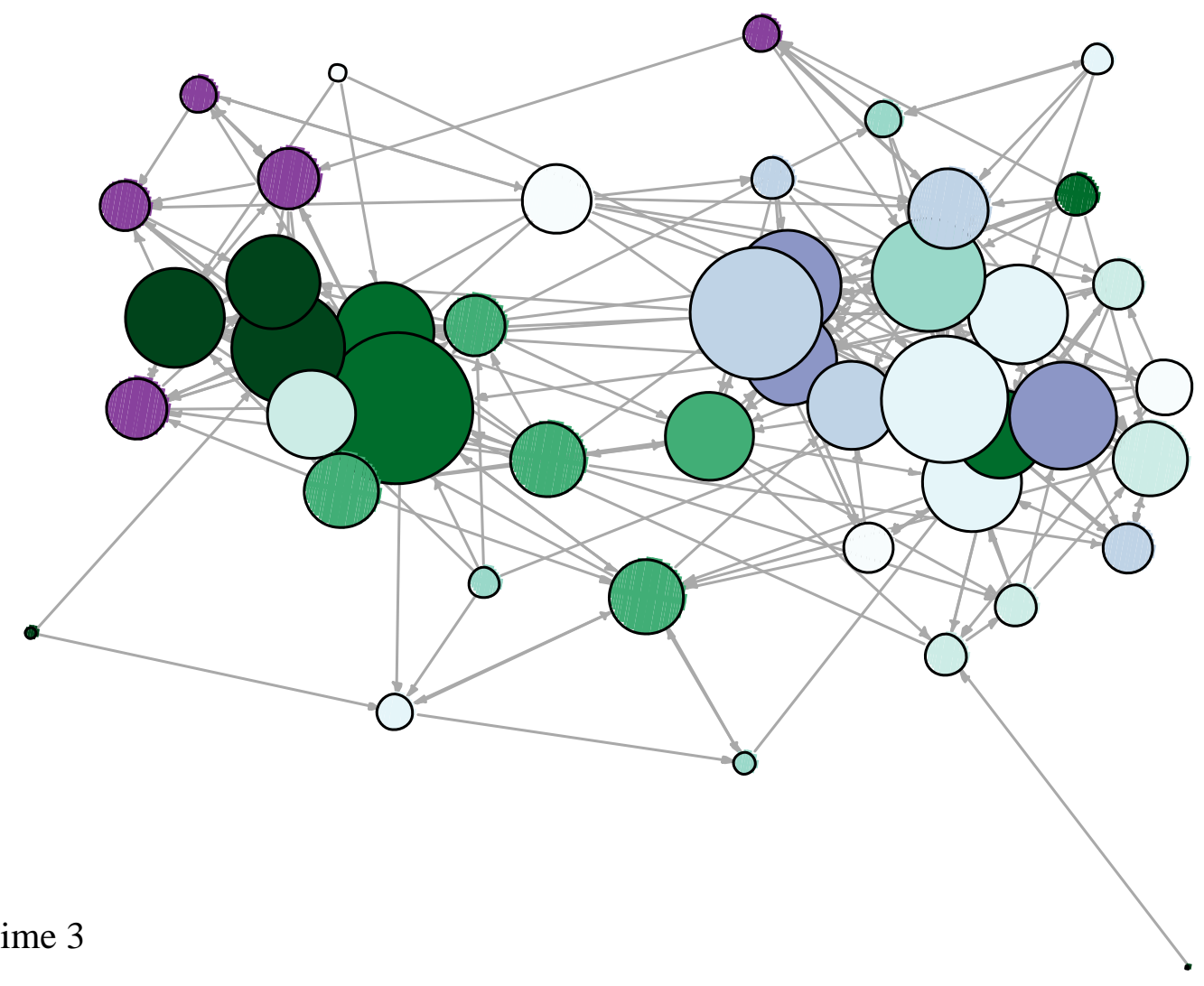


Figure 2. Change in YMCA network over time. Node size represents degree (how many friends a child has), while color represents activity level (dark purple is lowest, gray in the middle, dark green is highest)

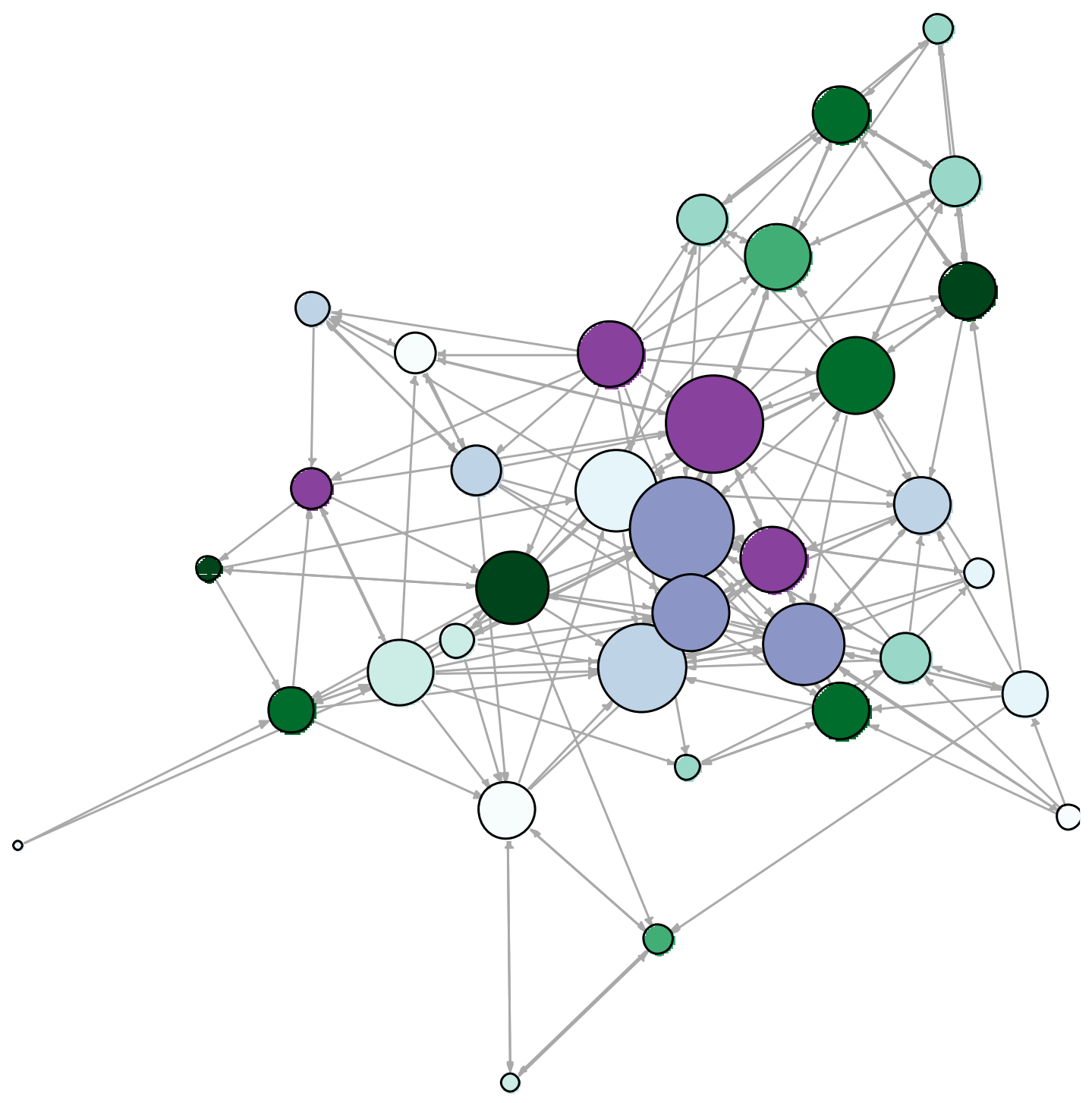

Time 1 


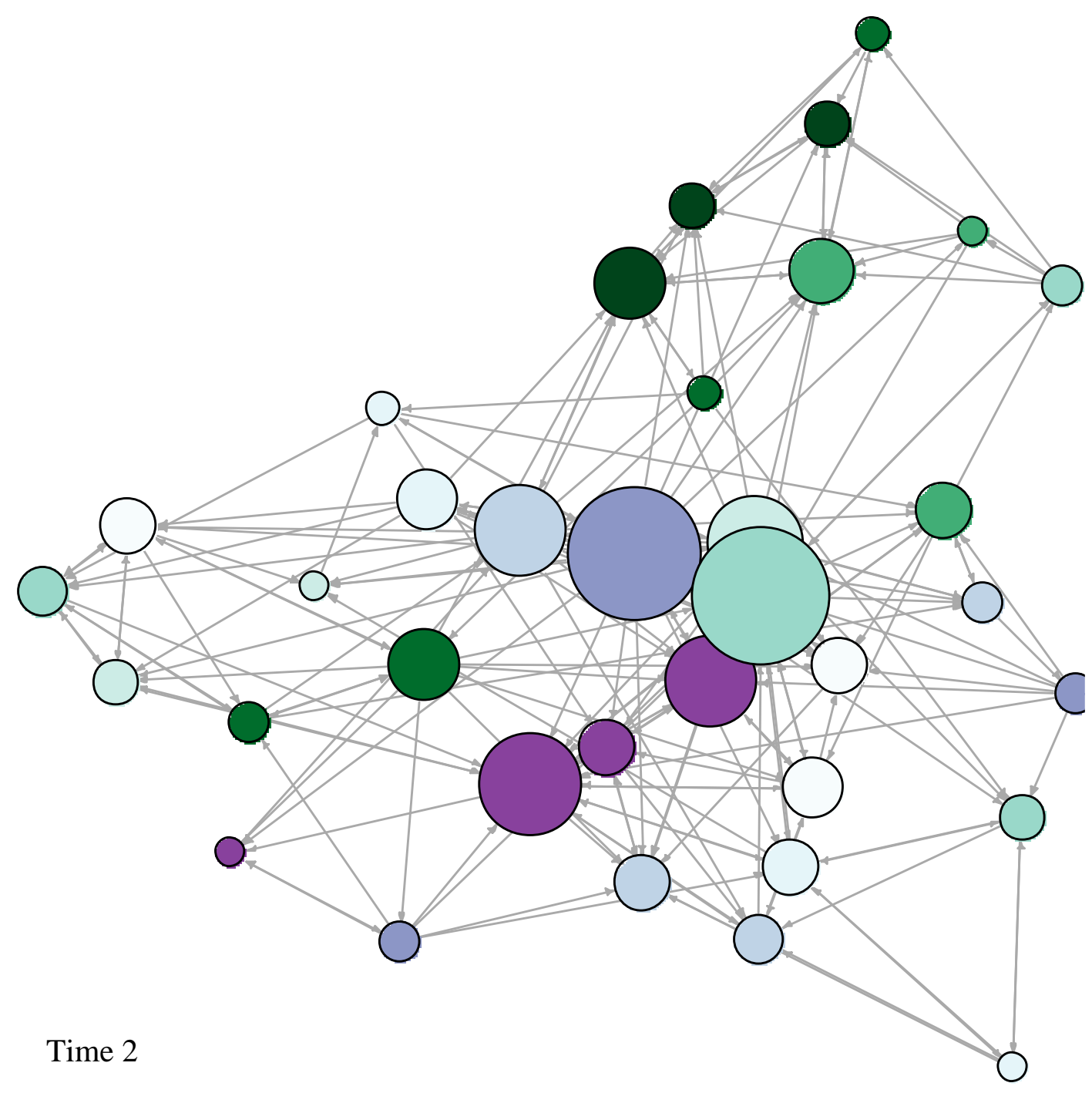




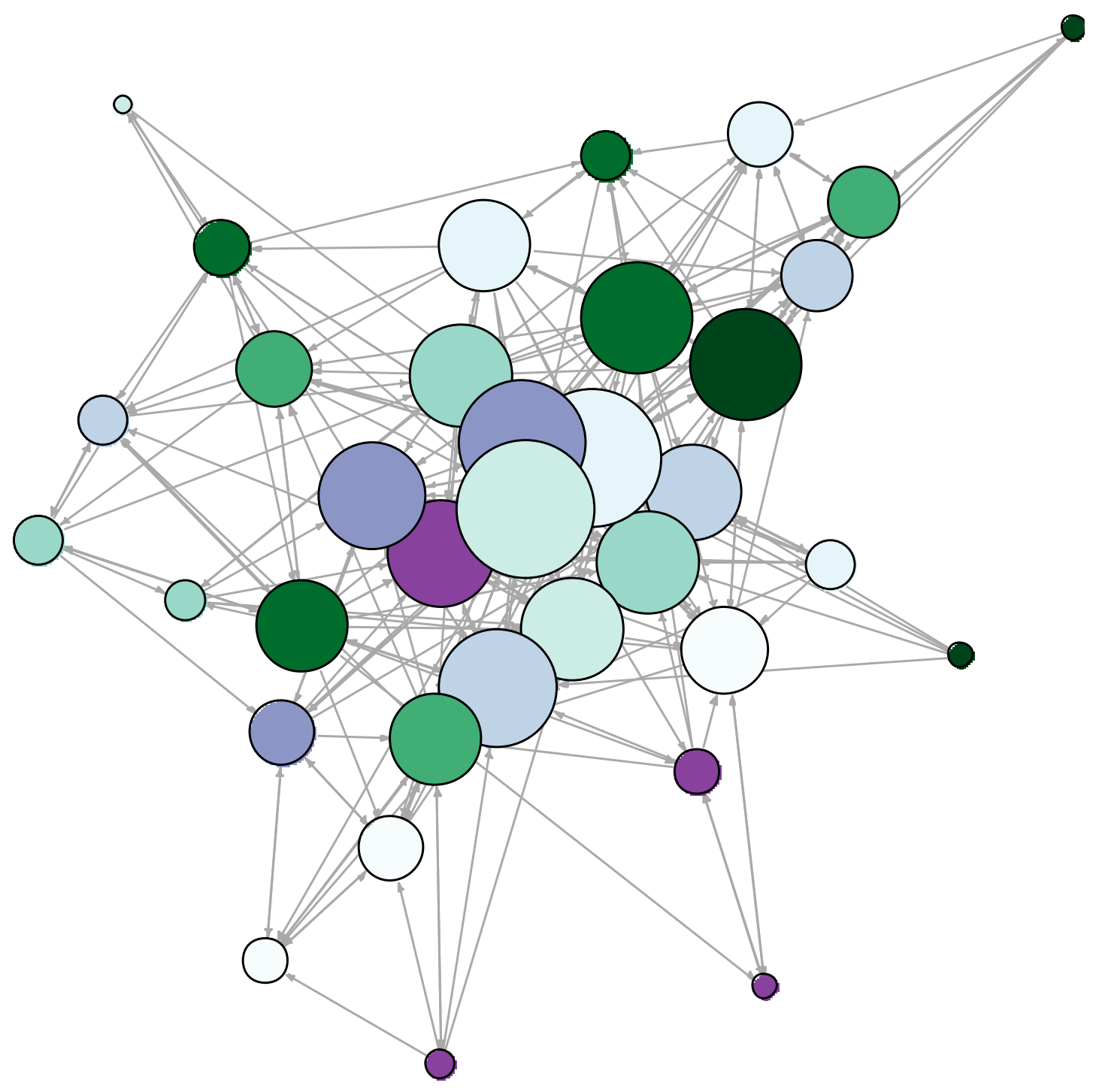

Time 3 


\section{Appendix 2}

I was not able to explore development of a dynamic social network model in my thesis work, though this is an issue I would like to explore further, possibly in my dissertation. The models I deployed lacked information on how an individual moves through a network over time because they were not built to examine those questions. Instead, they examine what could be causing network changes, rather than the changes themselves. Some dynamic network models have been formulated for use with large networks in computer science, such as Sarkar and Moore's (2006) or Xu and Zheng's (2009) network models using latent space modeling. Further development of these models is important for future work in dynamic social network analysis and should be adapted for use with small networks, such as the after school care networks used in this study.

One idea is to base a dynamic network model on the work of Chung, Walls, and Park (2007), who added covariates to a latent transition model. A child's probability of having a tie (friendship) with another child (or a group of children) based on various covariates could be conceptualized as a class and leaving that friendship/class and becoming friends with another child (or group of children) could be seen as a transition. Classes could be created based on other relevant covariates in the dataset, such as activity level, age, or gender. Latent transition models allow us to estimate the probability of class membership and transition between classes at a given time point simultaneously using Markov chain Monte Carlo (MCMC) estimation methods (Chung, Walls, \& Park, 2007). 
Alternatively, a binomial model could be explored where not having relationships with other people is coded as "failure" and having friendship ties with others is coded as "success," with the stability of ties viewed as desirable. A limitation of this conceptualization is that it inherently values having relationships in the network and lacks information to address other value-driven relationship questions (e.g., if a child is considered a "good" child with which to be friends). Exploring development of these types of model is important because they are longitudinal but also personspecific and would add to a growing literature of modeling network change over time in a unique way. 


\section{Appendix 3}

There are important differences between SAOMs and STERGMs in terms of modeling and assumptions underlying the modeling process. First, and most superficially, SAOMs are actor-based while STERGMs are dyad-based, with a focus on ties between actors (Block, Stadtfeld, \& Snijders, 2017). This difference is important because it creates a difference in the interpretation of the relative probability of a tie between the two models; parameters are interpreted at the graph level in STERGMs, whereas they are nested within individuals in SAOMs. STERGMs are also more parsimonious than SAOMs because they do not include the consideration of the individual actor as a focal point and maker of change in the graph (Block et al., 2017).

In SAOMs, outgoing potential ties are embedded within the actor's local structure, whereas in STERGMs, potential ties are considered in isolation (e.g., if my nearby neighbor becomes more popular I am not more likely to become friends with them than I am to someone else in the network who becomes more popular because the focus is not on the individual but on the graph as a whole). Block and colleagues (2017) conducted studies with cross-sectional networks showing that modeling effects in the same network using ERGMs and SAOMs can result in different parameter estimates.

Also important are the ways in which the two models use time. STERGMs are more sensitive to the length of time between network measurements because they are discrete time models, where tie changes within a time step are considered conditionally independent from one another. This differs from SAOMs, which are 
continuous time models and lack a conditional dependence assumption, meaning a change in ties between dyads immediately affects other dyads within the network within a time step. In SAOMs all parameter estimates (except for the rate parameter) are independent of the length of time between network measurements, in contrast to STERGMs, which experience an increase in magnitude of within-time effects and a decrease in magnitude in between-time effects as the time between network measurements increases (Schaefer \& Marcum, 2017).

Conditional independence is also influential in how the models are run and how the parameters are estimated. The conditional independence of ties in STERGMs puts the focus on local configurations within the network, with nodes that are distant in the network essentially conditionally independent from one another, because they are not close in the social space (Block et al., 2017). On the other hand, SAOMs assume that ties within a network can only change one at a time and actors choose which of their outgoing ties they will change (or choose not to change), which can be influenced by potential changes to their local configuration, similar to the STERGM reliance on local configurations, but with a focus on the individual (Block et al., 2017).

To take a closer look at the importance of independence versus dependence in tie formation, Block and colleagues (2017) used toy examples to show that using reciprocity as a parameter in ERGMs and SAOMs results in greater tie dependence in SAOMs, compared to ERGMs (findings that generalize to STERGMs, which are an extension of ERGMs). This is because a negative reciprocity parameter in an SAOM makes non-reciprocal ties more likely (the more incoming ties an individual has, the 
higher the probability is of that individual forming a non-reciprocal tie) and a positive reciprocity parameter makes them less likely, whereas the directionality of the reciprocity parameter does not affect non-reciprocal tie formation in ERGMs. They also show that there is a difference in outdegree distributions between the two models, which could impact outdegree parameter estimates when using the models in empirical research (Block et al., 2017).

The models do have some similarities. However, these similarities are not enough to overlook the stark differences between the models and careful consideration of which model to use is advised. Both models include a linear predictor, similar to those used in the generalized linear model tradition. The difference between the predictors is that the predictor for STERGMs is defined for the graph as a whole, while the predictor is defined for the individual actors in SAOMs. The linear predictor for ERGMs is:

$$
\sum_{k} \theta_{k} z_{k}(x)
$$

where $\theta_{\mathrm{k}}$ is a statistical parameter, similar to those in the generalized linear model, and where $\mathrm{z}_{\mathrm{k}}(\mathrm{x})$ are network statistics (Block et al., 2017). The linear predictor for SAOMs, given for node $i$ is as follows:

$$
\sum_{k} \beta_{k} s_{k, i}(x)
$$

where $\beta_{\mathrm{k}}$ is a statistical parameter, similar to those in the generalized linear model, and $\mathrm{S}_{\mathrm{k}, \mathrm{i}}(\mathrm{x})$ are network statistics with regard to node $i$ specifically (Block et al., 2017). 
The models are also similar in terms of how they model the probability of a tie changing, using Markov chain estimation (Block et al., 2017). An ERGM's probability distribution for random graphs, $\mathrm{X}$, can be written as:

$$
P_{\text {ERGM }}(X=x ; \theta)=\frac{\exp \left(\sum_{k} \theta_{k} z_{k}(x)\right)}{\kappa},
$$

which you will recognize as the exponential of the linear predictor over $\mathrm{k}$, the normalizing constant (Block et al., 2017). Unlike the ERGM probability distribution, the random probability distribution of SAOMs can't be expressed in closed form:

$$
P_{\mathrm{SAOM}}(x ; \beta)=\sum_{(h, l)} P_{\mathrm{SAOM}}\left(x^{ \pm h l} ; \beta\right) \cdot p_{\mathrm{SAOM}}\left(x^{ \pm h l} \rightarrow x ; \beta\right),
$$

with variables $h$ and $l$ are over all dyads within the network, $\beta$ as the statistical parameter, similar to generalized linear models, and $\mathrm{x}$ as the observed network (Block et al., 2017).

When considering ERGMs at the tie-level, the equation representing network transition from the observed network, $\mathrm{x}$, to the next network state, $\mathrm{x}^{ \pm \mathrm{ij}}$, is as follows:

$$
\begin{aligned}
p_{\text {ERGM }}\left(x \rightarrow x^{ \pm i j} ; \theta\right) & =\frac{1}{N(N-1)} \cdot \frac{\exp \left(\sum_{k} \theta_{k} z_{k}\left(x^{ \pm i j}\right)\right)}{\exp \left(\sum_{k} \theta_{k} z_{k}(x)\right)+\exp \left(\sum_{k} \theta_{k} z_{k}\left(x^{ \pm i j}\right)\right)} \\
& =\underbrace{\frac{1}{N(N-1)}}_{\text {select tie } x_{i j}} \cdot \underbrace{\frac{\exp \left(\sum_{k} \theta_{k} \Delta z_{k}\left(x, x^{ \pm i j}\right)\right)}{1+\exp \left(\sum_{k} \theta_{k} \Delta z_{k}\left(x, x^{ \pm i j}\right)\right)}}_{\text {change tie } x_{i j}},
\end{aligned}
$$

with the first factor, representing the probability of randomly selecting the ordered pair $(i, j)$, which is simply 1 divided by the product of the total number of nodes in the 
network, $N$, and one less than the total number of nodes, $N-1$. The second factor is the probability of changing the $(i, j)$ tie, rather than not changing it. The $\Delta \mathrm{z}_{\mathrm{k}}$ term is the change statistic (Block et al., 2017). For SAOMs, the probability of transition between the observed network, $\mathrm{x}$, and the subsequent network state, $\mathrm{x}^{ \pm \mathrm{ij}}$, given the local perspective of an actor, defined as the probability of node $i$ changing a tie (or non-tie) state with node $j$, is written as follows:

$$
\begin{aligned}
p_{\mathrm{SAOM}}\left(x \rightarrow x^{ \pm i j} ; \beta\right) & =\rho_{i} \times \frac{\exp \left(\sum_{k} \beta_{k} s_{k, i}\left(x^{ \pm i j}\right)\right)}{\sum_{h} \exp \left(\sum_{k} \beta_{k} s_{k, i}\left(x^{ \pm i h}\right)\right)} \\
& =\underbrace{\rho_{i}}_{\text {select actor } i} \times \underbrace{\frac{\exp \left(\sum_{k} \beta_{k} \Delta s_{k, i}\left(x, x^{ \pm i j}\right)\right)}{1+\sum_{h^{\prime}} \exp \left(\sum_{k} \beta_{k} \Delta s_{k, i}\left(x, x^{ \pm i h^{\prime}}\right)\right)}}_{\text {actor } i \text { changing tie } \pm i j},
\end{aligned}
$$

with the first term, $\rho_{i}$, as the probability that node $i$ gets to make a tie change, rather than any other node in the network, also called the rate function. The second term is the probability that node $i$ changes its tie with node $j$, rather than with any other node in the network. The change statistic for SAOMs is represented by $s_{k, i}\left(\mathrm{x}, \mathrm{x}^{ \pm \mathrm{ij}}\right)$ (Block et al., 2017). Together, these equations illustrate the difference in model estimation between ERGMs and SAOMs. 


\section{BIBLIOGRAPHY}

Anderson, A., Locke, J., Kretzmann, M., \& Kasari, C. (2016). Social network analysis of children with autism spectrum disorder: Predictors of fragmentation and connectivity in elementary school classrooms. Autism, 20(6), 700-709.

Block, P., Stadtfeld, C., \& Snijders, T. A. (2017). Forms of Dependence: Comparing SAOMs and ERGMs from Basic Principles. Sociological Methods \& Research, 1-57.

Chung, H., Walls, T. A., \& Park, Y. (2007). A latent transition model with logistic regression. Psychometrika, 72(3), 413-435.

Cooc, N., \& Kim, J. S. (2017). Peer influence on children's reading skills: A social network analysis of elementary school classrooms. Journal of Educational Psychology, 109(5), 727-761.

DeLay, D., Ha, T., Van Ryzin, M., Winter, C., \& Dishion, T. J. (2016). Changing friend selection in middle school: A social network analysis of a randomized intervention study designed to prevent adolescent problem behavior. Prevention Science, 17(3), 285-294.

Eccles, J. S., Wigfield, A., \& Schiefele, U. (1998). Motivation to succeed. In W. Damon \& N. Eisenberg (Eds.), Handbook of Child Psychology: Volume 3. Social, Emotional, and Personality Development (5 ${ }^{\text {th }}$ ed., p. 1017-1095). New York: Wiley.

Feiring, C., \& Lewis, M. (1989). The social networks of girls and boys from early through middle childhood. In D. Belle (Ed.), Children's Social Networks and Social Supports, 119-150. 
Gesell, S. B., Tesdahl, E., \& Ruchman, E. (2012). The distribution of physical activity in an after-school friendship network. Pediatrics, 129, 1064-71.

Golemiec, M., Schneider, J., Boyce, W. T., Bush, N. R., Adler, N., \& Levine, J. D. (2016). Layered social network analysis reveals complex relationships in kindergarteners. Frontiers in Psychology, 7, 276-282.

Greenhoot, A. F. (2011). Retrospective methods in developmental science. In B. Larsen, T. D., Little, \& N. A. Card (Eds.) Handbook of developmental research methods, 1, 196-210.

Handcock, M., Hunter, D., Butts, C., Goodreau, S., Krivitsky, P., \& Morris, M. (2017). ergm: Fit, simulate and diagnose exponential-family models for networks, The Statnet Project (<URL: http://www.statnet.org>). R package version 3.6.1.

Kindermann, T. A. (2007). Effects of naturally existing peer groups on changes in academic engagement in a cohort of sixth graders. Child Development, 78(4), 1186-1203.

Kindermann, T. A., DeLay, D., Laursen, B., Clark-Shim, H., \& Yelverton, R. (2013) Convergent findings about peer group influences on classroom engagement in a small town from analyses using RSiena and SEM. Poster presented at the biennial meetings of the Society for Research in Child Development. Seattle, WA.

Kolaczyk, E. D., \& Csárdi, G. (2014). Statistical analysis of network data with R. New York: Springer. 
Kolaczyk, E. D., \& Krivitsky, P. N., (2015). On the question of effective sample size in network modelling: An asymptotic inquiry, Statistical Science: A Review Journal of the Institute of Mathematical Statistics, 30(2), 184-198.

Krivitsky, P. N., \& Handcock, M. S. (2014). A separable model for dynamic networks. Journal of the Royal Statistical Society: Series B (Statistical Methodology), 76(1), 29-46.

Krivitsky, P., \& Handcock, M. (2016). Stergm: Fit, simulate, and diagnose models for network evolution base on exponential-family random graph models, The Statnet Project (<URL: http://www.statnet.org>). R package version 3.4.0.

Laninga-Wijnen, L., Ryan, A. M., Harakeh, Z., Shin, H., \& Vollebergh, W. A. (2017). The moderating role of popular peers' achievement goals in 5th-and 6thgraders' achievement-related friendships: A social network analysis. Journal of Educational Psychology, 1-19.

Leung, M-C. (1996). Social networks and self enhancement in Chinese children: A comparison of self reports and peer reports of group membership. Social Development, 5(2), 146-157.

Marsden, P. V. (2011). Survey methods for network data. In P. Carrington \& J. Scott (Eds.) The SAGE Handbook of Social Network Analysis, (p. 370-388). Thousand Oaks, CA: SAGE.

R Core Team (2016). R: A language and environment for statistical computing. $R$ Foundation for Statistical Computing, Vienna, Austria. URL https://www.Rproject.org/. 
Ripley, R., Boitmanis, K., Snijders, T. A. B., \& Schoenenberger, F. (2017). RSiena: Siena - simulation investigation for empirical network analysis. R package version 1.2-3. URL <https://CRAN.R-project.org/package=RSiena>

Ripley, R. M., Snijders, T. A., \& Preciado, P. (2011). Manual for RSIENA. University of Oxford, Department of Statistics, Nuffield College, 1-234.

Sarkar, P., \& Moore, A. W. (2006). Dynamic social network analysis using latent space models. In Advances in Neural Information Processing Systems (p. 1145-1152).

Schaefer, D. R., \& Marcum, C. S. (2017). Modeling Network Dynamics. SocArXiv, 139.

Schafer, J. L., \& Graham, J. W. (2002). Missing data: Our view of the state of the art. Psychological Methods, 7(2), 147-177.

Snijders, T. A. B., van de Bunt, G. G., \& Steglich, C. E. G. (2010). Introduction to stochastic actor-based models for network dynamics. Social Networks, 32, 4460.

Valente, T. W., Fujimoto, K., Chou, C. P., \& Spruijt-Metz, D. (2009). Adolescent affiliations and adiposity: A social network analysis of friendships and obesity. The Journal of Adolescent Health, 45(2), 202-204.

Wasserman, S., \& Faust, K. (1994). Social network analysis: Methods and applications. Cambridge, UK: Cambridge University Press.

Xu, A., \& Zheng, X. (2009). Dynamic social network analysis using latent space model and an integrated clustering algorithm. In Dependable, Autonomic and 
Secure Computing, 2009. DASC'09. Eighth IEEE International Conference (p. 620-625). 